\title{
Extremal density for sparse minors and subdivisions
}

\author{
John Haslegrave ${ }^{1}$, Jaehoon Kim ${ }^{1, *}$ and Hong Liu ${ }^{2}$ \\ ${ }^{1}$ Mathematics Institute, University of Warwick, Coventry CV4 7AL, UK \\ and ${ }^{2}$ Department of Mathematicsl Sciences, Daejeon 34141, South Korea \\ *Correspondence to be sent to: e-mail: jaehoon.kim@kaist.ac.kr
}

We prove an asymptotically tight bound on the extremal density guaranteeing subdivisions of bounded-degree bipartite graphs with a mild separability condition. As corollaries, we answer several questions of Reed and Wood on embedding sparse minors. Among others,

- $\quad(1+o(1)) t^{2}$ average degree is sufficient to force the $t \times t$ grid as a topological minor;

- $\quad(3 / 2+o(1)) t$ average degree forces every $t$-vertex planar graph as a minor, and the constant $3 / 2$ is optimal, furthermore, surprisingly, the value is the same for $t$-vertex graphs embeddable on any fixed surface;

- a universal bound of $(2+o(1)) t$ on average degree forcing every $t$-vertex graph in any nontrivial minor-closed family as a minor, and the constant 2 is best possible by considering graphs with given treewidth.

\section{Introduction}

Classical extremal graph theory studies sufficient conditions forcing the appearance of substructures. A seminal result of this type is the Erdoős-Stone-Simonovits theorem $[10,11]$, determining the asymptotics of the average degree needed for subgraph containment. It reads as

$$
d_{\supseteq}(H):=\lim _{n \rightarrow \infty} \inf \{c:|G| \geq n \text { and } d(G) \geq c|G| \Rightarrow G \supseteq H\}=1-\frac{1}{\chi(H)-1}
$$


where $\chi(H)$ is the chromatic number of $H$. We are interested here in the analogous problem of average degree conditions forcing $H$ as a minor. A graph $H$ is a minor of $G$, denoted by $G \succ H$, if it can be obtained from $G$ by vertex deletions, edge deletions and contractions.

The study of such problems has a long history. An initial motivation was Hadwiger's conjecture that every graph of chromatic number $t$ has $K_{t}$ as a minor, which is a far-reaching generalisation of the four-colour theorem. Since every graph of chromatic number $k$ contains a subgraph of average degree at least $k-1, a$ natural angle of attack is to find bounds on the average degree which will guarantee a $K_{t}$-minor. The first upper bound for general $t$ was given by Mader [22, 23], who subsequently improved this bound to $O(t \log t)$. In celebrated work of Kostochka [20] and, independently, Thomason [38], it was improved to the best possible bound $O(t \sqrt{\log t})$, Thomason subsequently determining the asymptotic [39]. Denoting

$$
d_{\succ}(H):=\inf \{c: d(G) \geq c \Rightarrow G \succ H\}
$$

he proved that $d_{\succ}\left(K_{t}\right)=\left(\alpha+o_{t}(1)\right) t \sqrt{\log t}$, where $\alpha=0.6382 \ldots$ is explicitly defined. This remained the best order of magnitude bound even for the chromatic number question until very recent breakthrough by Norin, Postle, and Song [29] and by Postle [30].

For more general minors, Myers and Thomason [26] resolved the problem when $H$ is polynomially dense, that is, having $|H|^{1+\Omega(1)}$ edges, showing that $d_{\succ}(H)=(\alpha \gamma(H)+$ $o(1))|H| \sqrt{\log |H|}$ for $\alpha$ as above and some explicitly defined $\gamma(H)$. However, for sparse graphs, their results only give $d_{\succ}(H)=o(|H| \sqrt{\log |H|})$, similar to the way that the Erdoős-Stone-Simonovits theorem only gives $d_{\supseteq}(H)=0$ for bipartite $H$, and so it is natural to ask for stronger bounds in this regime.

Reed and Wood [31] considered sparser graphs and, in particular, showed that for sufficiently large average degree $d(H)$, we have $d_{\succ}(H)<3.895|H| \sqrt{\log d(H)}$. They also obtain bounds linear in $e(H)$, which are better in the very sparse case of bounded average degree. Reed and Wood asked several interesting questions about the asymptotics of $d_{\succ}(H)$ for sparse $H$. Among sparse graphs, grids play a central role in graph minor theory $[6,32,36]$. Indeed, Reed and Wood raised the question of determining $d_{\succ}\left(\mathrm{G}_{t, t}\right)$, where $\mathrm{G}_{t, t}$ is the $t \times t$ grid. That is, what is the minimum $\beta>0$ such that every graph with average degree at least $\beta t^{2}$ contains $\mathrm{G}_{t, t}$ as a minor. Trivially $\beta \geq 1$ in order for the graph to have enough vertices, and their results give a bound of $\beta \leq 6.929$. 
This question provides the motivating example for our results. However, we shall focus on a special class of minors: subdivisions or topological minors. A subdivision of $H$ is a graph obtained from subdividing edges of $H$ to pairwise internally disjoint paths. The name of topological minor comes from its key role in topological graph theory. A cornerstone result in this area is Kuratowski's theorem from 1930 that a graph is planar if and only if it does not contain a subdivision of $K_{5}$ or $K_{3,3}$. Again, it is natural to ask what average degree $d$ will force $K_{t}$ as a topological minor, and we define analogously

$$
d_{\top}(H):=\inf \{c: d(G) \geq c \Rightarrow G \text { contains } H \text { as a topological minor }\}
$$

Clearly, for any $H, d_{\succ}(H) \leq d_{\top}(H)$. By considering complete bipartite graphs it is easy to see that $d_{\top}\left(K_{t}\right)=\Omega\left(t^{2}\right)$. Komlós and Szemerédi [19] and, independently, Bollobás and Thomason [2] gave a matching upper bound of $d_{\top}\left(K_{t}\right)=O\left(t^{2}\right)$. Note that clique topological minors are harder to guarantee than clique minors, as evidenced by the significant gap between this result and that of Kostochka and of Thomason for the latter. Furthermore, the leading coefficient of the quadratic bound on $d_{\top}\left(K_{t}\right)$ is still unknown. Much less is known for bounds on average degree guaranteeing sparse graphs as topological minors.

\subsection{Main result}

We study in this paper the problem of finding subdivisions of a natural class of sparse bipartite graphs. In particular, our main result offers the asymptotics of the average degree needed to force subdivisions of such graphs, showing that a necessary bound is already sufficient. It reads as follows.

Theorem 1.1. For given $\varepsilon>0$ and $\Delta \in \mathbb{N}$, there exist $\alpha_{0}$ and $d_{0}$ satisfying the following for all $0<\alpha<\alpha_{0}$ and $d \geq d_{0}$. If $H$ is an $\alpha$-separable bipartite graph with at most $(1-\varepsilon) d$ vertices and $\Delta(H) \leq \Delta$, and $G$ is a graph with average degree at least $d$, then $G$ contains a subdivision of $H$.

Here, a graph $H$ is $\alpha$-separable if there exists a set $S$ of at most $\alpha|H|$ vertices such that every component of $H-S$ has at most $\alpha|H|$ vertices. Graphs in many well-known classes are $o(1)$-separable. For example, any large graphs in nontrivial minor-closed family of graphs is $o(1)$-separable $[1,24]$. 
As an immediate corollary, our main result answers the above question of Reed and Wood in a strong sense by showing that any $\beta>1$ is sufficient to force the $k$ dimensional grid $\mathrm{G}_{t, \ldots, t}^{k}$ not only as a minor but as a topological minor, and so

$$
d_{\mathrm{\top}}\left(\mathrm{G}_{t, \ldots, t}^{k}\right)=d_{\succ}\left(\mathrm{G}_{t, \ldots, t}^{k}\right)=\left(1+o_{t}(1)\right) t^{k}
$$

We remark that the optimal constant 1 in Theorem 1.1 is no longer sufficient if $H$ is not bipartite. Indeed, if for example $H$ is the disjoint union of triangles, then the Corrádi-Hajnal theorem [5] implies that $d_{\succ}(H)=\frac{4}{3}|H|-2$. We shall elaborate more on the leading constant in the next section.

Our proof utilises both pseudorandomness from Szemerédi's regularity lemma and expansions for sparse graphs. The particular expander that we shall make use of is an extension of the one introduced by Komlós and Szemerédi, which has played an important role in some recent developments on sparse graph embedding problems, see for example, [14, 21].

Organisation. Applications of our main result will be given in Section 2. Preliminaries on expanders and basic building blocks are given in Section 3. In Section 4, we outline the strategies for proving Theorem 1.1. Its proof is then given in Sections 5-7. Lastly, concluding remarks and some open problems are given in Section 8.

\section{Applications}

Reed and Wood [31] raised several interesting questions on the average degree needed to force certain sparse graphs as minors. As corollaries of our main results, we answer many of these questions, and some others, with asymptotically optimal bounds.

\subsection{Planar graphs}

Problem A [31]. What is the least constant $c>0$ such that every graph with average degree at least $c t$ contains every planar graph with $t$ vertices as a minor?

Since a planar graph has average degree at most 6 , their results imply that $c \leq 14.602$. We can deduce the asymptotic answer to their question; in fact, rather surprisingly, the value is the same for graphs of any fixed genus, not just planar graphs. We find it convenient to use the following notation. The above problem basically asks for the maximum of $d_{\succ}(F)$ over all graphs in some family $\mathcal{F}$ with at most $t$ vertices. 
Define

$$
d_{\succ}(\mathcal{F}, t):=\inf \{c: d(G) \geq c \Rightarrow G \succ H, \forall H \in \mathcal{F} \text { with }|H| \leq t\}
$$

Theorem 2.1. For any $\varepsilon>0$ and any $g \geq 0$, there exists $t_{0}$ such that for every $t \geq t_{0}$ the following hold:

- every graph with average degree at least $(3 / 2+\varepsilon) t$ contains every graph of genus at most $g$ with $t$ vertices as a minor, but

- there exists a graph of average degree $3 t / 2-O(1)$, which does not contain every planar graph with $t$ vertices as a minor.

That is, writing $\mathcal{F}_{g}$ for the family of all graphs with genus $g$, we have $d_{\succ}\left(\mathcal{F}_{g}, t\right)=$ $(3 / 2+o(1)) t$.

Proof. We first prove the second statement. Take any planar graph $H^{*}$ on $t$ vertices containing $\lfloor t / 4\rfloor$ disjoint copies of $K_{4}$. It is easy to verify that $K_{2, n}$ is a series-parallel graph for any $n$, and so does not contain $K_{4}$ as a minor (see [8]). Therefore, any bipartite graph that does contain $K_{4}$ as a minor is not a subgraph of $K_{2, n}$ for any $n$, and so must have at least 3 vertices in each part. If $G$ is a bipartite graph that contains $H^{*}$ as a minor, then $G$ must contain at least 3 vertices in each part for each of the $\lfloor t / 4\rfloor$ copies of $K_{4}$, so must contain at least $3\lfloor t / 4\rfloor$ vertices in each part. Therefore, the complete bipartite graph with parts of order $3\lfloor t / 4\rfloor-1$ and $n$ does not contain $H^{*}$ as a minor. This graph has average degree at least $6\lfloor t / 4\rfloor-3$ if $n$ is sufficiently large.

Next, we deduce the first statement from Theorem 1.1. It is sufficient to prove the case $\varepsilon<1$. Let $\Delta=\lfloor 20 / \varepsilon\rfloor \geq 20$, and fix $\alpha \leq \alpha_{0}(\varepsilon / 2, \Delta)$. Let $H$ be an arbitrary $t$-vertex graph of genus at most $g$. If $H$ has a vertex of degree $k>\Delta$, replace it with two adjacent vertices of degrees $k-(\Delta-1)$ and $\Delta$; when doing this, allocate the neighbours of the original vertex to the two vertices in such a way as to preserve the genus. Continue until no vertices with degree bigger than $\Delta$ remain. Thus, we obtain a graph $H^{\prime}$ of genus at most $g$ with at most $t+\sum_{V \in V(H)} \frac{d(v)}{\Delta-1} \leq t+(6 t+O(1)) /(\Delta-1) \leq(1+\varepsilon / 3) t$ vertices which contains $H$ as a minor.

Let $A$ and $B$ be two independent sets in $H^{\prime}$, chosen so that $|A|+|B|$ is as large as possible. If $g=0$ (i.e., $H^{\prime}$ is planar), then considering the two largest classes of a four-colouring of $H^{\prime}$ shows that $|A|+|B| \geq\left|H^{\prime}\right| / 2$. If $g>0$, using a result of Djidjev [7], we may find a planar induced subgraph on $\left|H^{\prime}\right|-O\left(\sqrt{\left|H^{\prime}\right|}\right)$ vertices, and considering a four-colouring of this subgraph shows that $|A|+|B| \geq\left|H^{\prime}\right| / 2-o(t)$. So, in either case, we 
have $\left|V\left(H^{\prime}\right) \backslash(A \cup B)\right| \leq(1+\varepsilon) t / 2$ for $t$ sufficiently large. Note that our choice of $A$ and $B$ ensures that every other vertex has neighbours in both $A$ and $B$.

We define a bipartite graph $H^{\prime \prime}$ as follows. Vertices in $A \cup B$, and edges between them, are unchanged. For each vertex $V \in V\left(H^{\prime}\right) \backslash(A \cup B), H^{\prime \prime}$ has two vertices $V_{A^{\prime}}, V_{B}$ with an edge between them. Every edge of $H^{\prime}$ of the form $u v$ with $u \in A$ and $v \in V\left(H^{\prime}\right) \backslash(A \cup B)$ becomes an edge $u v_{B}$, and every edge of the form $u v$ with $u \in B$ and $v \in V\left(H^{\prime}\right) \backslash(A \cup B)$ becomes an edge $u v_{A}$. For every edge $v w$ with $v, w \in V\left(H^{\prime}\right) \backslash(A \cup B)$, choose an ordering $V, W$ arbitrarily and add the edge $V_{A} W_{B}$. In the resulting graph $H^{\prime \prime}$, every vertex has degree at most that of the corresponding vertex in $H^{\prime}$, and, by contracting every edge of the form $v_{A} v_{B}, H^{\prime \prime}$ contains $H^{\prime}$ as a minor.

The genus of $H^{\prime \prime}$ may be greater than $g$, but the bounded genus of $H^{\prime}$ ensures that $H^{\prime}$ is $\alpha / 2$-separable for $t$ sufficiently large. Since any subset of $V\left(H^{\prime}\right)$ of size at most $\alpha\left|H^{\prime}\right| / 2$ corresponds to a subset of $V\left(H^{\prime \prime}\right)$ of size at most $\alpha\left|H^{\prime \prime}\right|, H^{\prime \prime}$ is $\alpha$-separable. Now, $H^{\prime \prime}$ is a bipartite graph with at most $(3 / 2+\varepsilon) t$ vertices, so by Theorem 1.1 , we can find a subdivision of $H^{\prime \prime}$, which in turn contains $H$ as a minor, in any graph with average degree at least $(3 / 2+\varepsilon) t$ provided $t$ is sufficiently large.

\subsection{A universal bound for nontrivial minor-closed families}

Many important classes of graphs are naturally closed under taking minors, for example, graphs embeddable on a given surface considered in Theorem 2.1. The seminal graph minor theorem of Robertson and Seymour (proved in a sequence of papers culminating in [34]) shows that every minor-closed family can be characterised by a finite list of minimal forbidden minors. For example, the linklessly embeddable graphs are defined by a minimal family of seven forbidden minors, including $K_{6}$ and the Petersen graph [37]. The existence of a forbidden minor characterisation has farreaching algorithmic implications, since for any fixed graph $F$ there exists an algorithm to determine whether an $n$-vertex graph contains $F$ as a minor in $O\left(n^{3}\right)$ time [33], and hence there is a cubic-time algorithm (since improved to quadratic [13]) to check for membership of any given minor-closed family; prior to these results, it was not even known that the property of having a linkless embedding was decidable. However, the constants concealed by the asymptotic notation are typically prohibitively large. Furthermore, for many families a complete forbidden minor classification, and hence a specific algorithm, is not known, and the number of minimal forbidden minors can be extremely large, even for families that may be very naturally and simply defined. For example, there are over $68,000,000,000$ minimal forbidden minors for the class of $Y \Delta Y$-reducible graphs [41]. 
We can extend the methods of the previous subsection to minor-closed families more generally. For each $k \in \mathbb{N}$, define $\alpha_{k}(G):=\max \{|U|: U \subseteq V(G), \chi(G[U])=k\}$. So, $\alpha_{1}(G)$ is the usual independence number, and $\alpha_{2}(G)$ is the maximum size of the union of two independent sets.

Theorem 2.2. Let $\mathcal{F}$ be a nontrivial minor-closed family. For each $F \in \mathcal{F}$ with $t$ vertices, we have

$$
2 t-2 \alpha(F)-O(1) \leq d_{\succ}(F) \leq 2 t-\alpha_{2}(F)+o(t) .
$$

Proof. It is well known that the $t$-vertex graphs in $\mathcal{F}$ are $o(1)$-separable with at most $C_{\mathcal{F}} t$ edges for some constant $C_{\mathcal{F}}[1]$.

To prove the upper bound, take two disjoint independent sets $A$ and $B$ with $\alpha_{2}(F)=|A \cup B|$. By the same argument as in Theorem 2.1, we can define a bipartite graph $F^{\prime \prime}$ containing $F$ as a minor with $2 t-\alpha_{2}(F)+o(t)$ vertices having bounded maximum degree. Apply Theorem 1.1 to $F^{\prime \prime}$ to obtain the upper bound.

To prove the lower bound, consider $K_{s, n-s}$ where $s=t-\alpha(F)-1$. If it contains an $F$-minor, let $V_{1}, \ldots, V_{|F|}$ be the vertex sets corresponding to the vertices of $F$. By our choice of $s$, it is easy to see that at least $\alpha(F)+1$ of them have to completely reside in the independent set of size $n-s$ in $K_{s, n-s}$, which is impossible. Thus, $K_{s, n-s}$ does not contain an $F$-minor. By choosing large $n$, we have $d\left(K_{s, n-s}\right) \geq 2 t-2 \alpha(F)-O(1)$.

Theorem 2.2 yields the following universal bound for all nontrivial minor-closed families.

Corollary 2.3. For any nontrivial minor-closed family $\mathcal{F}$, we have

$$
d_{\succ}(\mathcal{F}, t) \leq(2+o(1)) t
$$

We remark that the constant 2 above cannot be improved as we shall see in Corollary 2.4 .

\subsection{Graphs with given treewidth or clique minor}

Reed and Wood asked the following questions for specific minor-closed families.

Problem B [31]. What is the least function $g_{1}$ such that every graph with average degree at least $g_{1}(k) \cdot t$ contains every graph with $t$ vertices and treewidth at most $k$ as a minor? 
Problem C [31]. What is the least function $g_{2}$ such that every graph with average degree at least $g_{2}(k) \cdot t$ contains every $K_{k}$-minor-free graph with $t$ vertices as a minor?

Graphs with treewidth at most $k$ are $k$-degenerate, and hence have at most $k t$ edges, and graphs without a $K_{k}$-minor have average degree $O(k \sqrt{\log k})$. Consequently, the result of Reed and Wood [31] showed that $g_{i}(k)=O(\sqrt{\log k})$ for $i \in\{1,2\}$. As a corollary of Theorem 2.2, we get the following optimal bound of $g_{i}(k)=2+o_{k}(1)$, showing that somewhat surprisingly, when $k$ is sufficiently large, both the treewidth and the size of a largest clique minor play negligible roles in the leading coefficient.

Corollary 2.4. $\quad$ Every graph with average degree $\left(2+o_{k}(1)\right) t$ contains every graph with $t$ vertices, which either has treewidth at most $k$ or is $K_{k}$-minor-free, as a minor.

Proof. The upper bound follows immediately from Theorem 2.2. Note that a disjoint union of copies of $K_{k+1}$ has treewidth $k$. Then, the unbalanced complete bipartite graph $K_{(1-1 /(k+1)) t-1, n}$ provides the matching lower bound $2(1-1 /(k+1)) t=\left(2+o_{k}(1)\right) t$.

For graphs without $K_{k}$-minor, consider instead a disjoint union of copies of $K_{k-1}$.

\subsection{Beyond minor-closed classes}

In Section 2.2, the two properties of minor-closed families that we needed were $o(1)$ separability and bounded average degree. Many other sparse graph classes have these properties. In particular, any class which obeys a strongly sublinear separator theorem is $o(1)$-separable, see [24].

A $k$-shallow minor of a graph $G$ is a minor for which each contracted subgraph has radius at most $k$. We say that a graph class $\mathcal{C}$ has bounded expansion if the average degree of $k$-shallow minors of graphs in $\mathcal{C}$ is bounded by a function of $k$; in particular, since 0 -shallow minors are just subgraphs, $\mathcal{C}$ itself has bounded average degree. If the bound is a polynomial function, we say that $\mathcal{C}$ has polynomial expansion. These definitions were introduced by Nešetřil and Ossona de Mendez [27].

Classes of polynomial expansion have strongly sublinear separator theorems, and for hereditary classes the two notions are equivalent [9]. Thus, we may extend Theorem 2.2 to classes of polynomial expansion. Such classes include the 1-planar graphs, that is, the graphs which may be embedded in the plane with each edge crossing at most one other edge once [28], and intersection graphs of systems of balls with only a bounded number meeting any point [25]. 
Polynomial expansion is a much weaker property than being minor-closed. It is easy to see, for example, that any graph can be suitably subdivided to obtain a 1-planar graph. However, Borodin [3] showed that all 1-planar graphs are 6-colourable, and since they include disjoint unions of $K_{6}$, we obtain the following tight result from the extension of Theorem 2.2 to classes of polynomial expansion.

Corollary 2.5. The class $\mathcal{P}_{1}$ of 1 -planar graphs satisfies $d_{\succ}\left(\mathcal{P}_{1}, t\right)=\left(5 / 3+o_{t}(1)\right) t$.

\section{Preliminaries}

For $n \in \mathbb{N}$, let $[n]:=\{1, \ldots, n\}$. Given a set $X$ and $k \in \mathbb{N}$, let $\left(\begin{array}{l}X \\ k\end{array}\right)$ be the family of all $k$-sets in $X$. For brevity, we write $v$ for a singleton set $\{v\}$ and $x y$ for an unordered pair $\{x, y\}$. If we claim that a result holds whenever we have $0<a \ll b, c \ll d<1$, it means that there exist positive functions $f, g$ such that the result holds as long as $a<f(b, c)$ and $b<g(d)$ and $c<g(d)$. We will not compute these functions explicitly. In many cases, we treat large numbers as if they were integers, by omitting floors and ceilings if it does not affect the argument. We write log for the base-e logarithm.

\subsection{Graph notation}

Given graphs $H$ and $G$, and a copy of an $H$-subdivision in $G$, we call the vertices that correspond to $V(H)$ the anchor vertices of the subdivision. For a given path $P=x_{1} \ldots x_{t}$, we write $\operatorname{Int}(P)=\left\{x_{2}, \ldots, x_{t-1}\right\}$ to denote the set of its internal vertices. Given a graph $H$, a set of vertices $S \subseteq V(H)$ and a set of edges $F \subseteq E(H)$, denote by $H-S=H[V(H) \backslash S]$ the subgraph induced on $V(H) \backslash S$ and by $H-F$ the spanning subgraph obtained from $H$ by removing edges in $F$.

Given a graph $G$, denote its average degree $2 e(G) /|G|$ by $d(G)$. For two sets $X, Y \subseteq$ $V(G)$, the (graph) distance between them is the length of a shortest path from $X$ to $Y$. For two graphs $G, H$, we write $G \cup H$ to denote the graph with vertex set $V(G) \cup V(H)$ and edge set $E(G) \cup E(H)$. A $k$-star denotes a copy of $K_{1, k}$, which is a star with $k$ edges. Given a collection of graphs $\mathcal{F}=\left\{F_{i}: i \in I\right\}$, we write $V(\mathcal{F})=\bigcup_{i \in I} V\left(F_{i}\right)$ and $|\mathcal{F}|=|I|$. For path $P$ and a vertex set $U$, we write $\left.P\right|_{U}$ for the induced subgraph of $P$ on vertex set $V(P) \cap U$. We say that $G$ is a graph with a vertex partition $V_{1}, \ldots, V_{s}$ if $V(G)=V_{1} \cup \cdots \cup V_{s}$ and $V_{1}, \ldots, V_{s}$ are pairwise disjoint.

For a set of vertices $X \subseteq V(G)$ and $i \in \mathbb{N}$, denote by

$$
N^{i}(X):=\{u \in V(G): \text { the distance between } X \text { and } u \text { is exactly } i\}
$$


the $i$ th sphere around $X$, and write $N^{0}(X)=X, N(X):=N^{1}(X)$, and for $i \in \mathbb{N} \cup\{0\}$, let $B^{i}(X)=\bigcup_{j=0}^{i} N^{j}(X)$ be the ball of radius $i$ around $X$. We write $\partial(X)$ for the edge-boundary of $X$, that is, the set of edges between $X$ and $V(G) \backslash X$ in $G$. Given another set $Z \subseteq V(G)$, we write $N(X, Z)=N(X) \cap Z$ for the set of neighbours of $X$ in $Z$.

\subsection{Robust expander}

To define the robust graph expansion, we need the following function. For $\varepsilon_{1}>0$ and $t>0$, let $\rho(x)$ be the function

$$
\rho(x)=\rho\left(x, \varepsilon_{1}, t\right):= \begin{cases}0 & \text { if } x<t / 5 \\ \varepsilon_{1} / \log ^{2}(15 x / t) & \text { if } x \geq t / 5\end{cases}
$$

where, when it is clear from context, we will not write the dependency on $\varepsilon_{1}$ and $t$ of $\rho(x)$. Note that when $x \geq t / 2, \rho(x)$ is decreasing, while $\rho(x) \cdot x$ is increasing.

Komlós and Szemerédi $[18,19]$ introduced a notion of expander $G$ in which any set $X$ of reasonable size expands by a sublinear factor, that is, $\left|N_{G}(X)\right| \geq \rho(|X|)|X|$. We shall extend this notion to a robust one such that similar expansion occurs even after removing a relatively small set of edges.

Definition 3.1. $\quad\left(\varepsilon_{1}, t\right)$-robust-expander: A graph $G$ is an $\left(\varepsilon_{1}, t\right)$-robust-expander if for every subset $X \subseteq V(G)$ of size $t / 2 \leq|X| \leq|G| / 2$, and every subset $F \subseteq E(G)$ with $|F| \leq$ $d(G) \cdot \rho(|X|)|X|$, we have

$$
\left|N_{G-F}(X)\right| \geq \rho(|X|)|X|
$$

We shall use the following version of expander lemma, stating that every graph contains a robust expander subgraph with almost the same average degree.

Lemma 3.2. Let $C>30, \varepsilon_{1} \leq 1 /(10 C), \varepsilon_{2}<1 / 2, d>0$ and $\rho(x)=\rho\left(x, \varepsilon_{1}, \varepsilon_{2} d\right)$ as in (1). Then every graph $G$ with $d(G)=d$ has a subgraph $H$ such that

- $H$ is an $\left(\varepsilon_{1}, \varepsilon_{2} d\right)$-robust-expander;

- $d(H) \geq(1-\delta) d$, where $\delta:=\frac{C \varepsilon_{1}}{\log 3}$;

- $\delta(H) \geq d(H) / 2$;

- $H$ is $v d$-connected, where $v:=\frac{\varepsilon_{1}}{6 \log ^{2}\left(5 / \varepsilon_{2}\right)}$. 
We remark that, though almost retaining the average degree, the robust expander subgraph $H$ in Lemma 3.2 could be much smaller than $G$. For instance, if $G$ is a union of many vertex disjoint small cliques, then $H$ could be just one of those cliques. This drawback often makes it difficult to utilise expanders iteratively within graphs. We include the proof of the robust expander lemma, Lemma 3.2, in the appendix of the online version [12].

A key property of the robust expanders that we shall use is that they have small (logarithmic) diameter.

Lemma 3.3. [19] Corollary 2.3. If $G$ is an $n$-vertex $\left(\varepsilon_{1}, t\right)$-robust-expander, then for any two vertex sets $X_{1}, X_{2}$ each of size at least $x \geq t / 2$, and a vertex set $W$ of size at most $\rho(x) x / 4$, there exists a path in $G-W$ between $X_{1}$ and $X_{2}$ of length at most

$$
\frac{2}{\varepsilon_{1}} \log ^{3}\left(\frac{15 n}{t}\right) .
$$

\subsection{Exponential growth for small sets}

In an $\left(\varepsilon_{1}, t\right)$-robust-expander graph, for a set $X$ with size at least $t / 2$, the ball $B^{i}(X)$ grows with the radius $i$. For our purpose, we need to quantify how resilient this growth is to deletion of some thin set around $X$.

Definition 3.4. For a set $X \subseteq W$ of vertices, the paths $P_{1}, \ldots, P_{q}$ are consecutive shortest paths from $X$ within $W$ if the following holds. For each $i \in[q],\left.P_{i}\right|_{W}$ is a shortest path from $X$ to some vertex $v_{i} \in W \backslash X$ in the graph restricted to $W \backslash \bigcup_{j \in[i-1]} V\left(P_{j}\right)$.

In particular, the following proposition shows that the rate of expansion for small sets is almost exponential in a robust expander even after deleting a few consecutive shortest paths.

Proposition 3.5. Let $0<1 / d \ll \varepsilon_{1}, \varepsilon_{2} \ll 1$. Suppose $G$ is an $n$-vertex $\left(\varepsilon_{1}, \varepsilon_{2} d\right)$-robustexpander and $X, Y$ are disjoint sets of vertices with $|X|=X \geq \varepsilon_{2} d$ and $|Y| \leq \frac{1}{4} \cdot \rho(x) \cdot X$. Let $P_{1}, \ldots, P_{q}$ be consecutive shortest paths in $G-Y$ from $X$ within $B_{G-Y}^{r}(X)$, where $1 \leq r \leq \log n$ and $q<x \log ^{-8} x$, and let $P=\bigcup_{i \in[q]} V\left(P_{i}\right)$. Then for each $i \in[r]$, we have

$$
\left|B_{G-P-Y}^{i}(X \backslash P)\right| \geq \exp \left(i^{1 / 4}\right) .
$$


Proof. For each $i \geq 0$, let $Z_{i}=B_{G-P-Y}^{i}(X \backslash P)$. As $P_{1}, \ldots, P_{q}$ are consecutive shortest paths from $X$, for each $i \geq 0$, each path $P_{j}, j \in[q]$, can intersect with the set $N_{G-Y}\left(Z_{i}\right)$ on at most $i+2$ vertices. Indeed, otherwise, we can replace the initial segment of $P_{j}$ with a path in $Z_{i} \cup N_{G-Y}\left(Z_{i}\right)$ of length $i+1$ to get a shorter path in $G-Y-\bigcup_{k \in[j-1]} V\left(P_{k}\right)$, contradicting the choice of $P_{j}$. Thus, $\left|N_{G-Y}\left(Z_{i}\right) \cap P\right| \leq(i+2) q$. Consequently, the expansion of $G$ implies for each $i \geq 0$ that

$$
\begin{aligned}
\left|Z_{i+l}\right| & =\left|Z_{i}\right|+\left|N_{G}\left(Z_{i}\right) \backslash(Y \cup P)\right| \geq\left|Z_{i}\right|+\rho\left(\left|Z_{i}\right|\right)\left|Z_{i}\right|-|Y|-\left|N_{G-Y}\left(Z_{i}\right) \cap P\right| \\
& \geq\left|Z_{i}\right|+\frac{3}{4} \rho\left(\left|Z_{i}\right|\right)\left|Z_{i}\right|-(i+2) q .
\end{aligned}
$$

Let

$$
f(z)=\exp \left(z^{1 / 4}\right) \quad \text { and } \quad g(z):=x+\frac{1}{2} \rho(x) x z .
$$

We first use induction on $i$ to show that for each $1 \leq i \leq \log ^{4} x,\left|Z_{i}\right| \geq g(i)$. Since $X$ contains one vertex from each path, $\left|Z_{0}\right|=x-q$. Applying (2) with $i=0$ gives

$$
\left|Z_{1}\right| \geq x-q+\frac{3}{4} \rho(x-q)(x-q)-2 q \geq x+\frac{3}{4} x \rho(x)-4 q,
$$

and since $q<x \log ^{-8} x$, this is at least $x+\frac{1}{2} x \rho(x)$ as required.

Now, for $1 \leq i \leq \log ^{4} x$, we use (2) together with the induction hypothesis and the facts that $\rho(z) z$ is increasing when $z \geq x$ and $\frac{1}{4} \rho(x) x \geq \frac{(i+2) x}{\log ^{8} x}>(i+2) q$ to obtain

$$
\left|Z_{i+l}\right| \geq\left|Z_{i}\right|+\frac{3}{4} \rho\left(\left|Z_{i}\right|\right)\left|Z_{i}\right|-(i+2) q \geq\left|Z_{i}\right|+\frac{1}{2} \rho(x) x=\left|Z_{i}\right|+g(i+1)-g(i) \geq g(i+1) .
$$

We may then assume $i>\log ^{4} x$, as $f(i) \leq g(i) \leq\left|Z_{i}\right|$ when $i \leq \log ^{4} x$. Now, as $i>\log ^{4} x, \frac{f(i)}{i^{7 / 4}} \geq \frac{f\left(\log ^{4} x\right)}{\left(\log ^{4} x\right)^{7 / 4}}=\frac{x}{\log ^{7} x}$ and so 


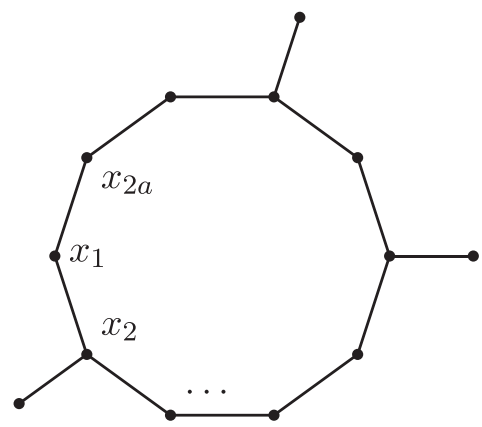

Fig. 1. A $(2 a, b)$-sun with $a=5$ and $b=3$.

$$
(i+2) q<i \cdot \frac{2 x}{\log ^{8} x} \leq i \cdot \frac{f(i)}{i^{7 / 4}}=\frac{f(i)}{i^{3 / 4}}
$$

Also note that $f(i+1)-f(i) \leq \frac{f(i)}{i^{3 / 4}}$ and $\rho\left(\left|Z_{i}\right|\right)\left|Z_{i}\right| \geq \rho(f(i)) f(i) \geq \frac{\varepsilon_{1} f(i)}{i^{1 / 2}}$. Thus, we have

$$
\begin{aligned}
\left|Z_{i+l}\right| & \geq\left|Z_{i}\right|+\frac{3}{4} \rho\left(\left|Z_{i}\right|\right)\left|Z_{i}\right|-(i+2) q \geq\left|Z_{i}\right|+\frac{3 \varepsilon_{1} f(i)}{4 i^{1 / 2}}-\frac{f(i)}{i^{3 / 4}} \\
& \geq\left|Z_{i}\right|+\frac{f(i)}{i^{3 / 4}} \geq\left|Z_{i}\right|+f(i+1)-f(i) \geq f(i+1),
\end{aligned}
$$

as desired.

\subsection{Basic building structures}

The following structures will serve as basic building blocks for our constructions of subdivisions.

Definition 3.6. (2a,b)-Sun For integers $a \geq b \geq 0$, a $(2 a, b)$-sun is a bipartite graph consisting of a cycle $x_{1}, \ldots, x_{2 a}$ and leaves $y_{1}, \ldots, y_{b}$, where for each $i \in[a]$ the vertex $x_{2 i}$ is adjacent to at most one leaf, and for each $i \in[b]$ the leaf $y_{i}$ is adjacent to a vertex $x_{2 j}$ for some $j \in[a]$. See Figure 1 .

Note that a $(2 a, 0)$-sun is just an even cycle of length $2 a$.

Definition 3.7. $\quad\left(h_{1}, h_{2}, h_{3}\right)$-unit For $h_{1}, h_{2}, h_{3} \in \mathbb{N}$, an $\left(h_{1}, h_{2}, h_{3}\right)$-unit is a tree $F$ with a distinguished vertex $u$ (the core vertex of $F$ ), which consists of 


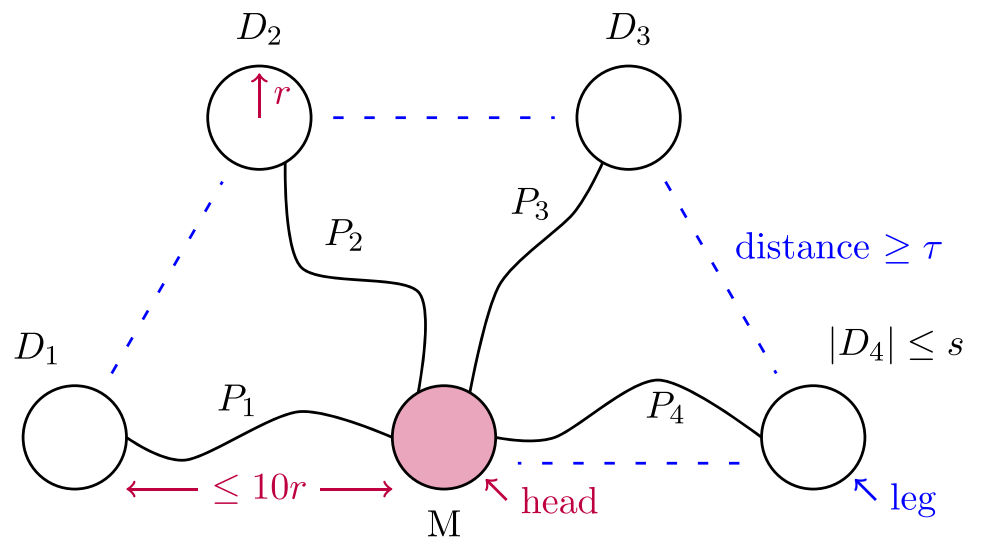

Fig. 2. A $(4, s, r, \tau)$-nakji (Definition 3.9).

- a collection of $h_{1}$ disjoint stars $\left\{S_{X_{i}}: i \in\left[h_{1}\right]\right\}$, with $S_{x_{i}}$ having central vertex $x_{i}$ and $h_{2}$ leaves, together with

- a collection $\left\{P_{i}: i \in\left[h_{1}\right]\right\}$ of paths from $u$ to $x_{i}$ respectively, each of length at most $h_{3}$, which are pairwise disjoint except at $u$.

Define the exterior $\operatorname{Ext}(F)$ to be the set of leaves of $F$, and the interior $\operatorname{lnt}(F):=V(F) \backslash$ $\operatorname{Ext}(F)$. For every vertex $w \in \operatorname{Ext}(F)$, let $P(F, W)$ be the unique path from the core vertex $u$ to $w$ in $F$.

Definition 3.8. $\quad\left(h_{0}, h_{1}, h_{2}, h_{3}\right)$-web For $h_{0}, h_{1}, h_{2}, h_{3} \in \mathbb{N}$, an $\left(h_{0}, h_{1}, h_{2}, h_{3}\right)$-web is a tree $W$ with a distinguished vertex $V$ (the core vertex of $W$ ), which consists of

- a collection of $h_{0}$ disjoint $\left(h_{1}, h_{2}, h_{3}\right)$-units $\left\{F_{u_{i}}: i \in\left[h_{0}\right]\right\}$, with $F_{u_{i}}$ having core vertex $u_{i}$, together with

- a collection $\left\{Q_{i}: i \in\left[h_{0}\right]\right\}$ of paths from $v$ to $u_{i}$ respectively, each of length at most $h_{3}$, which are pairwise disjoint except at $v$, and with each $Q_{i}$ disjoint from $\bigcup_{j \in\left[h_{0}\right]} V\left(F_{u_{j}}\right)$ except at $u_{i}$.

Define the exterior $\operatorname{Ext}(W):=\bigcup_{i \in\left[h_{0}\right]} \operatorname{Ext}\left(F_{u_{i}}\right)$, interior $\operatorname{Int}(W):=V(W) \backslash \operatorname{Ext}(W)$ and centre $\operatorname{Ctr}(W):=\bigcup_{i \in\left[h_{0}\right]} V\left(Q_{i}\right)$. For every vertex $w \in \operatorname{Ext}(W)$, let $P(W, w)$ be the unique path from the core vertex $v$ to $w$ in $W$. See Figure 3.

Definition 3.9. $\quad(t, s, r, \tau)$-nakji Given $t, s, r, \tau \in \mathbb{N}$, a graph $N$ is a $(t, s, r, \tau)$-nakji ${ }^{1}$ in $G$ if it contains vertex disjoint sets $M$ and $D_{1}, \ldots, D_{t}$, each having size at most $s$, and paths $P_{1}, \ldots, P_{t}$, such that for each $i \in[t]$ 


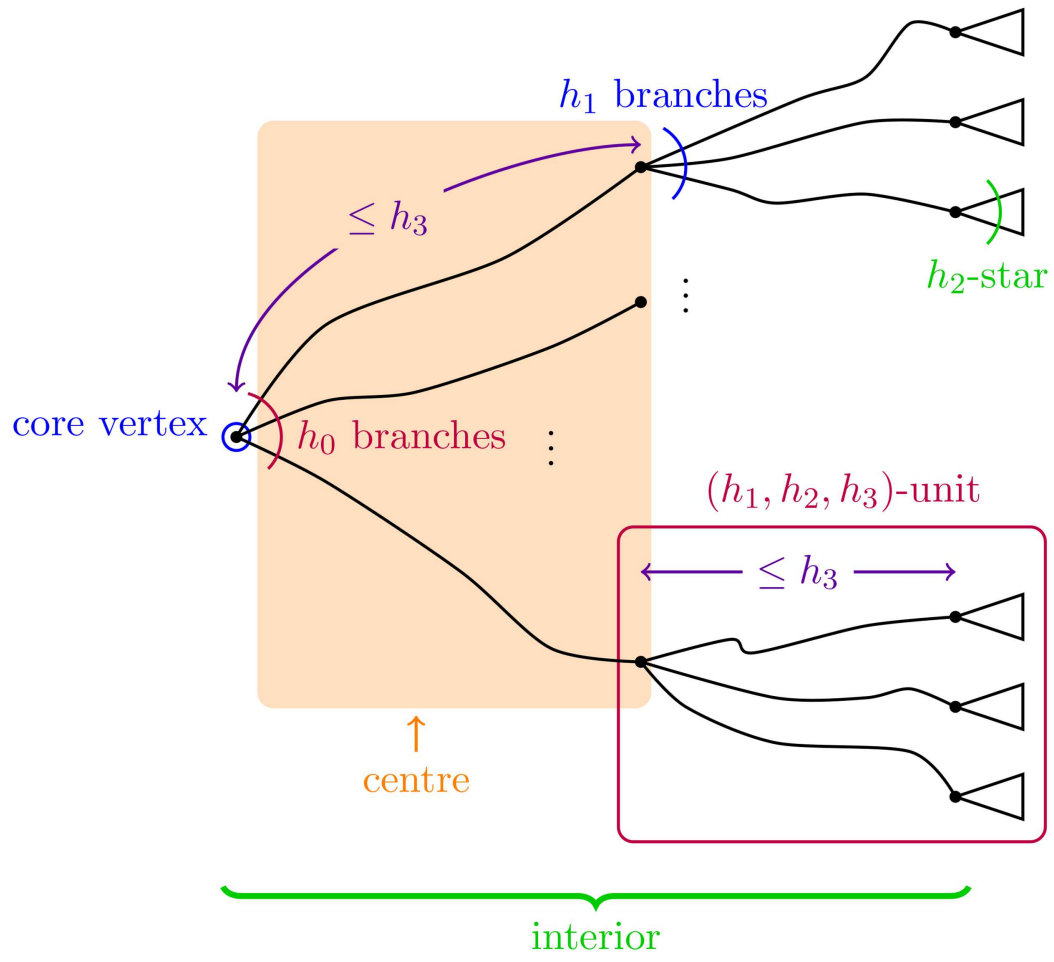

Fig. 3. An $\left(h_{0}, h_{1}, h_{2}, h_{3}\right)$-web.

- $P_{i}$ is an $M, D_{i}$-path with length at most $10 r$, and all paths $P_{i}, i \in[t]$, are pairwise internally disjoint;

- $D_{i}$ has diameter at most $r$, and all $D_{i}, i \in[t]$, are a distance at least $\tau$ in $G$ from each other and from $M$, and they are disjoint from internal vertices of $\bigcup_{i \in[t]} P_{i}$.

We call $M$ the head of the nakji and each $D_{i}, i \in[t]$, a leg. See Figure 2 .

\section{Outline of the Proofs}

To prove Theorem 1.1, we first use Lemma 3.2 to pass to a robust expander subgraph without losing much on the average degree. Depending on the density of the expander, we use different approaches. Roughly speaking, when the expander has positive edge density, we will utilise pseudorandomness via the machinery of the graph regularity lemma and the blow-up lemma (Lemma 4.1), whereas if the expander is not dense, then we exploit its sublinear expansion property (Lemmas 4.2 and 4.3). 
Lemma 4.1. Let $0<1 / d, \alpha \ll \delta, \eta, 1 / \Delta<1$ and $\delta \ll \varepsilon$, let $H$ be a graph with at most $(1-\varepsilon) d$ vertices such that $\Delta(H) \leq \Delta$, and let $G$ be an $n$-vertex graph. Suppose that $d>\eta n$ and $H$ is bipartite and $\alpha$-separable. If $d(G) \geq(1-\delta) d$, then $G$ contains $H$ as a subgraph.

Lemma 4.2. Let $0<1 / d \ll \eta \ll \varepsilon_{1}, \varepsilon_{2} \ll \varepsilon, 1 / \Delta<1$, let $H$ be a graph with at most $(1-\varepsilon) d$ vertices such that $\Delta(H) \leq \Delta$, let $G$ be an $n$-vertex graph, and write $m=$

$\frac{2}{\varepsilon_{1}} \log ^{3}\left(\frac{15 n}{\varepsilon_{2} d}\right)$. Suppose $G$ is an $\left(\varepsilon_{1}, \varepsilon_{2} d\right)$-robust-expander with $d(G) \geq \varepsilon^{2} d$. If $m^{100} \leq d \leq$ $\eta n$, then $G$ contains an $H$-subdivision.

Lemma 4.3. Let $0<1 / d \ll v, \delta \ll \varepsilon_{1}, \varepsilon_{2} \ll \varepsilon, 1 / \Delta<1$, let $H$ be a graph with at most ( $1-$ $\varepsilon) d$ vertices such that $\Delta(H) \leq \Delta$, let $G$ be an $n$-vertex graph, and write $m=\frac{2}{\varepsilon_{1}} \log ^{3}\left(\frac{15 n}{\varepsilon_{2} d}\right)$. Suppose $H$ is bipartite and $G$ is an $\left(\varepsilon_{1}, \varepsilon_{2} d\right)$-robust-expander with $d(G) \geq(1-\delta) d$ and $\delta(G) \geq d(G) / 2$. If $d<m^{100}$, then $G$ contains an $H$-subdivision.

Our main theorem readily follows, assuming these three lemmas.

Proof. Proof of Theorem 1.1. For given $\varepsilon$, let $\eta, v, \delta, \varepsilon_{1}, \varepsilon_{2}, \alpha_{0}, 1 / d_{0}$ be small enough so that Lemmas 4.1-4.3 holds for all $\alpha \leq \alpha_{0}$ and $d>d_{0}$. We apply Lemma 3.2 to $G$ to obtain a subgraph $G^{\prime}$, which is an $n^{\prime}$-vertex $\left(\varepsilon_{1}, \varepsilon_{2} d\right)$-robust-expander and $d\left(G^{\prime}\right) \geq(1-\delta) d$.

If $d \geq \eta n^{\prime}$, then as $H$ is $\alpha$-separable, Lemma 4.1 implies that $H \subseteq G^{\prime}$. If $d<\eta n^{\prime}$, then Lemmas 4.2 and 4.3 imply that $G$ contains an $H$-subdivision. This proves the theorem.

In the rest of this section, we outline the ideas in our constructions. Let $G$ be an expander and $H$ be the bounded-degree bipartite graph whose subdivision we want to embed in $G$.

\subsection{Embeddings in dense graphs}

The regularity lemma essentially partitions our graph $G$ into a bounded number of parts, in which the bipartite subgraphs induced by most of the pairs of parts behave pseudorandomly. The information of this partition is then stored to a (weighted) fixedsize so-called reduced graph $R$ that inherits the density of $G$.

We seek to embed $H$ in $G$ using the blow-up lemma, which boils down to finding a 'balanced' bounded-degree homomorphic image of $H$ in $R$. This is where the additional separable assumption on $H$ kicks in. The separability of $H$ (in the form of 
sublinear bandwidth) enables us to cut $H$ into small pieces to offer suitable 'balanced' homomorphic images.

Now, if the reduced graph $R$ has chromatic number at least three, the density of $R$ inherited from $G$ is just large enough to guarantee an odd cycle long enough in $R$ to serve as our bounded-degree homomorphic image of $H$.

It is, however, possible that $R$ is a bipartite graph, hence all cycles within are even cycles, which might not be long enough for our purpose. Indeed, in the worst case scenario that $H$ is an extremely asymmetric bipartite graph, an even cycle has to be twice as long as an odd one to be useful. This is because in the odd cycle we can circumvent the asymmetry of $H$ by 'breaking the parity' via wrapping around the odd cycle twice.

To handle the case when $R$ is bipartite, instead of cycles, we will make use of the sun structure (Definition 3.6), in which the leaves attaching to the main body of the sun help in balancing out the asymmetry of $H$.

\subsection{Embeddings in robust expanders with medium density}

The robust expansion underpins all of our constructions of $H$-subdivisions when the graph $G$ is no longer dense. At a high level, in $G$, we anchor on some carefully chosen vertices and embed paths between anchors (corresponding to the edge set of $H$ ) one at a time.

As these paths in the subdivision need to be internally vertex disjoint, to realise this greedy approach, we will need to build a path avoiding a certain set of vertices. This set of vertices to avoid contains previous paths that we have already found and often some small set of 'fragile' vertices that we wish to keep free.

To carry out such robust connections, we use the small-diameter property of expanders (Lemma 3.3). Let $m$ be the diameter of $G$. Recall that $H$ is of order at most $d$ with bounded degree and we need to embed $e(H)=O(d)$ paths. Thus, all in all, the set of vertices involved in all connections, say $W$, is of size $O(d m)$. To enjoy Lemma 3.3, we want to anchor at vertices with large 'boundary' compared with $W$, that is, being able to access many ( $d m^{10}$ say) vertices within short distance.

With that being said, if there are now $d$ vertices of high degree (at least $d m^{10}$ ), we can easily finish the embedding anchoring on these high degree vertices. This almost enables us to view $G$ as if it is a 'relatively regular' graph. In reality, what happens is that we can assume the set of high degree vertices, $L$, is small: $|L|<d$; and deduce from 
the robust expansion property that $G-L$ is still dense. It is worth pointing out that this is where we need to extend the original notion of expander to this robust one.

Without high degree vertices, we turn to the web structure (Definition 3.8), in which the core vertex has a large 'boundary' (the web's exterior) of size about $d m^{9}$. If there are $d$ webs of suitable size, we can then anchor on their core vertices and connect pairs via the exteriors of the corresponding webs. One thing to be careful about is that a web will become useless if the (few) vertices in its centre are involved in previous connections. To prevent this, when constructing paths between exteriors, we protect the 'fragile' centres of all webs.

Lastly, as $G$ is 'relatively regular', we can pull out many large stars (size roughly d) and link them up to find the webs one by one. The construction of webs is one of the places we require $G$ to be not too sparse ( $d$ being a large power of $m$ suffices).

\subsection{Embeddings in sparse robust expanders}

The current way of building and connecting webs breaks down if the expander is too sparse, say with average degree at most $\log \log n$. We will have to rely on other structures to build subdivisions in this case.

Let us first look at the easier problem of finding $H$-minors, in which case we just need to find $d$ large balls (and contract them afterwards) and find $O(d)$ internally disjoint paths between them. Note that now $|L|<d \leq m^{100}$ is quite small. Suppose additionally that $G-L$ has average degree $\Omega(d)$ and within it we can find $d$ vertices, $v_{1}, \ldots, v_{d}$, pairwise a distance $\sqrt{\log n}$ apart, such that for each $V_{i}$, the ball $B_{i}$ of radius say $(\log \log n)^{20}$ around it has size at least $m^{200}$. So each $B_{i}$ is large enough to enjoy exponential growth (Proposition 3.5) avoiding all paths previously built. Now to get, say, a $v_{i}, v_{j}$-path, we first expand $B_{i}, B_{j}$ to larger balls with radius say $(\log n)^{1 / 10}$. These larger balls are so gigantic that we can connect them avoiding all the smaller balls $\bigcup_{i \in[d]} B_{i}$. It is left to find such $v_{i}$ and $B_{i}$. We can find them one by one, by collectively growing a set $U$ of pairwise far apart vertices past $L$ and using an averaging argument to locate the next $v_{i}$ that expands well in $G-L$.

Coming back to embedding $H$-subdivisions, we shall follow the general strategy as that of finding minors. However, an immediate obstacle we encounter is the following. To get a subdivision instead of a minor, we need to be able to lead up to $\Delta(H)=O(1)$ many paths arriving at $B_{i}$ disjointly to $v_{i}$. In other words, each anchor vertex $v_{i}$ has to expand even after removing $O(1)$ disjoint paths starting from itself. 
Here comes the problem: in the minor case, we just need to expand $U$ ignoring a smaller set $L$; whereas now $U$ is asked to expand past a larger set of $\Theta(|U|)$ vertices that are used in the previous connections. Our expansion property is simply too weak for this.

This is where the nakji structure (Definition 3.9) comes into play. It is designed precisely to circumvent this problem by doing everything in reverse order. Basically, instead of looking for anchor vertices that expand robustly, we rather anchor on nakjis and link them via their legs first and then extend the paths from the legs in each nakji's head using connectivity.

Why do we require that the head and legs of a nakji are stretched far apart? This is so that, before linking nakjis together to a subdivision, we can expand each leg without bumping into any other part to an enormous size, so that each connection made leaves irrelevant structures untouched.

The remaining task is then to find many nakjis in $G-L$. This is done essentially by linking small subexpanders within $G-L$. A subtlety here worth pointing out is that we only have $|L|<d$, while each of the subexpanders, though having size $\Omega(d)$, could be smaller than $L$. This keeps us from expanding and linking each subexpander in $G-L$. Intuitively, given that $L$ is not large, one would like to take a huge set of subexpanders, whose union is so large and thus grows easily past $L$. However, as the expanding function $\rho(\cdot)$ is sublinear, if there are too many subexpanders to begin with, after averaging, the expansion rate of each subexpander in $G-L$ is too weak to be useful. To overcome this difficulty, instead, we shall average over a set of subexpanders of appropriate size that is just big enough to ignore $L$ and on the other hand just small enough that $\rho(\cdot)$ does not decay too much.

\section{Separable Bipartite Graphs in Dense Graphs}

In this section, we prove Lemma 4.1. We will use Szemerédi's regularity lemma; for a detailed survey of this lemma and its numerous applications, see $[16,17]$. Let $d_{G}(A, B):=$ $\frac{e(G[A, B])}{|A||B|}$ be the density of a bipartite graph $G$ with vertex classes $A$ and $B$. For a positive number $\varepsilon>0$, we say that a bipartite graph $G$ with vertex classes $A$ and $B$ is $\varepsilon$-regular if every $X \subseteq A$ and $Y \subseteq B$ with $|X| \geq \varepsilon|A|$ and $|Y| \geq \varepsilon|B|$ satisfy $\left|d_{G}(A, B)-d_{G}(X, Y)\right| \leq \varepsilon$. We say that it is $\left(\varepsilon, \delta^{+}\right)$-regular if it is $\varepsilon$-regular and $d_{G}(A, B) \geq \delta$.

The following lemma is a version of the regularity lemma suitable for our purpose. The discussion before Theorem 1.10 in [17] shows that the following lemma can be easily derived from the usual regularity lemma. 
Lemma 5.1. Suppose $0<1 / n \ll 1 / r_{1} \ll \varepsilon \ll \tau<1$ with $n \in \mathbb{N}$ and let $G$ be an $n$-vertex graph with $d(G) \geq d n$ for some $d \in(0,1)$. Then, there is a partition of the vertex set of $G$ into $V_{1}, \ldots, V_{r}$ and a graph $R$ on the vertex set $[r]$ such that the following holds:

1. $1 / \varepsilon \leq r \leq r_{1}$;

2. for each $i \in[r]$, we have $\frac{(1-\varepsilon) n}{r} \leq\left|V_{i}\right| \leq \frac{(1+\varepsilon) n}{r}$;

3. $d(R) \geq(d-2 \tau) r$;

4. for all $i j \in E(R)$, the graph $G\left[V_{i}, V_{j}\right]$ is $\left(\varepsilon, \tau^{+}\right)$-regular.

The graph $R$ above is often called the reduced graph of $G$ (with respect to the partition $V_{1}, \ldots, V_{r}$ ). One of the reasons why the regularity lemma is useful is that it can be combined with the blow-up lemma of Komlós, Sárközy and Szemerédi (see [15, Remark 8]). Here, we only need the following weaker version of the blow-up lemma which only yields a non-spanning subgraph in a given graph.

Theorem 5.2. Suppose $0<1 / n \ll \varepsilon \ll \tau, 1 / \Delta<1$ and $1 / n \ll 1 / r$ with $r, n \in \mathbb{N}$. Suppose $H$ is a graph with $\Delta(H) \leq \Delta$ having a vertex partition $X_{1}, \ldots, X_{r}, G$ is a graph with a vertex partition $V_{1}, \ldots, V_{r}$, and $R$ is a graph on the vertex set $[r]$ with $\Delta(R) \leq \Delta$. Suppose further that the following hold.

1. For each $i \in[r], X_{i}$ is an independent set in $H$ and $\left|X_{i}\right| \leq\left(1-\varepsilon^{1 / 2}\right)\left|V_{i}\right|$.

2. For each $i j \in E(R)$, the graph $G\left[V_{i}, V_{j}\right]$ is $\left(\varepsilon, \tau^{+}\right)$-regular.

3. For each $\left(\begin{array}{c}\{i, j\} \in \\ {[r] 2}\end{array}\right)$ with $i j \notin E(R)$, the graph $H$ contains no edges from $X_{i}$ to $X_{j}$. Then $G$ contains $H$ as a subgraph.

\subsection{Balanced homomorphic image of $H$}

We now show how to find a suitable partition of $H$ to invoke the blow-up lemma. This will use the separability property; however, we find it more convenient to work with bandwidth. A graph $H$ has bandwidth $b$ if we can order its vertices $x_{1}, \ldots, x_{|H|}$ such that $x_{i} X_{j} \notin E(H)$ for $|i-j|>b$. In general, small bandwidth is a stronger notion than small separability. However, the following result of Böttcher, Pruessmann, Taraz and Würfl shows that for bounded-degree graphs the two notions are roughly equivalent.

Lemma 5.3. [4, Theorem 5] Suppose $0<1 / d \ll \alpha \ll \beta \ll 1 / \Delta \leq 1$. If $H$ is an $\alpha$ separable graph with at most $d$ vertices and $\Delta(H) \leq \Delta$, then $H$ has bandwidth at most $\beta d$. 
We want to partition $H$ into almost equal-sized sets $X_{1}, \ldots, X_{r}$ for an appropriate $r$ such that all edges of $H$ lie between two consecutive sets $X_{i}$ and $X_{i+1}$, where we write $X_{r+1}=X_{1}$. In other words, we want to find a 'balanced' homomorphism from $H$ into $C_{r}$.

Later we will apply the regularity lemma to $G$ and find a cycle $C$ in the reduced graph we obtain, then apply the blow-up lemma with the above partition of $H$ where $r=|C|$. If $|C|$ is odd, then the value $r$ is large enough for us to fit $H$ into $G\left[\bigcup_{i \in C} V_{i}\right]$. However, if $|C|$ is even and $H$ is an unbalanced bipartite graph, then that strategy does not work. For such a case, we need to consider a sun instead.

The following two lemmas provide partitions of $H$ suitable for our purpose. The first finds a 'balanced' homomorphism of $H$ into an odd cycle $C_{r}$ and the second finds a 'balanced' homomorphism of $H$ into a suitable sun. As the two proofs are similar, we omit the proof of the first lemma here. It will be available in the appendix of the online version [12].

Lemma 5.4. Suppose $0<1 / d \ll \beta \ll 1 / r, \delta \ll 1 / \Delta<1$ and $r$ is an odd integer. If $H$ is a bipartite graph with at most $(1-\delta) d$ vertices and bandwidth at most $\beta d$ satisfying $\Delta(H) \leq \Delta$, then we can find a partition $X_{1}, \ldots, X_{r}$ such that the following hold.

- For each $i \in[r]$, we have $\left|X_{i}\right| \leq \frac{d}{r}$.

- Every edge of $H$ is between $X_{i}$ and $X_{i+1}$ for some $i \in[r]$ (taking $X_{r+1}=X_{1}$ ).

Lemma 5.5. Suppose $1 / d \ll \beta \ll 1 / r, \delta \ll \varepsilon, 1 / \Delta \leq 1$ and $R_{0}$ is a $(2 s, q)$-sun with $r=s+q$ and $q \leq s$. Suppose that $H$ is a bipartite graph having at most $(1-\delta) d$ vertices and bandwidth at most $\beta d$, with $\Delta(H) \leq \Delta$. Then, we can find a partition $\left\{X_{u}: u \in V\left(R_{0}\right)\right\}$ of $V(H)$ as follows.

- For each $u \in V\left(R_{0}\right)$, we have $\left|X_{u}\right| \leq \frac{d}{r}$.

- Every edge of $H$ is between $X_{u}$ and $X_{V}$ for some $u v \in E\left(R_{0}\right)$.

Proof. As $H$ has bandwidth at most $\beta d$, there exists an ordering $x_{1}, \ldots, x_{|H|}$ of $V(H)$ such that $x_{i} x_{j} \in E(H)$ implies $|i-j| \leq \beta d$. Write $A \cup B$, where $|A| \geq|B|$, for the bipartition of $H$, and set $\gamma=|B| /|A|$; note that $1 / \Delta \leq \gamma \leq 1$. We label the vertices of $R_{0}$ as follows. Let $u_{1}, \ldots, u_{2 s}$ be the vertices of the cycle in order, let $p_{1}, \ldots, p_{q}$ be the indices for which $u_{p_{i}}$ has a leaf neighbour (where each $p_{i}$ is even), and let the leaf neighbour of $u_{p_{i}}$ be $v_{p_{i}}$ for each $i \in[q]$. We will first find a partition $\left\{X_{i}: i \in[r]\right\} \cup\left\{X_{p_{\ell}}^{\prime}: \ell \in[q]\right\}$ of $V(H)$ and later we convert this to a desired partition $\left\{X_{u}: u \in V\left(R_{0}\right)\right\}$. We consider all indices in the sun 
(respectively in the partition of $H$ ) up to the congruence modulo $2 s$ (respectively $r$ ), that is, $u_{a+2 s}=u_{a}$ and $X_{a+r}=X_{a}$ for all $a \in \mathbb{N}$.

Let $t=\left\lceil(2 \beta)^{-1 / 2}+1\right\rceil$. We divide the vertices of $H$ according to the ordering as follows: for each $i \in\left[t^{2}\right]$, let

$$
\begin{aligned}
& Y_{i}^{A}=\left\{x_{j} \in A:(2 i-3) \beta d<j \leq(2 i-1) \beta d\right\} \quad \text { and } \\
& Y_{i}^{B}=\left\{x_{j} \in B:(2 i-2) \beta d<j \leq 2 i \beta d\right\} .
\end{aligned}
$$

Note that this guarantees that no edge of $H$ is between $Y_{i}^{A}$ and $Y_{j}^{B}$ with $j \notin\{i-1, i\}$ and $V(H)=\bigcup_{i \in\left[t^{2}\right]}\left(Y_{i}^{A} \cup Y_{i}^{B}\right)$. For each $i \in[t]$ and $C \in\{A, B\}$, let

$$
W_{i}^{C}=\bigcup_{j \in[s]} Y_{(i-1) t+j}^{C} \quad \text { and } \quad Z_{i}^{C}=\bigcup_{j \in[t] \backslash[s]} Y_{(i-1) t+j}^{C}
$$

In the claim below, we will decide to which part $X_{\ell}$ we assign the vertices in $Z_{i}^{C}$. To make such an assignment possible while keeping the edges only between two consecutive parts, we allow the vertices in $W_{i}^{C}$ to be assigned to some other parts. As each set $W_{i}^{C}$ is much smaller than $Z_{i}^{C}$, the uncontrolled assignments of $W_{i}^{C}$ will not harm us too much. Indeed, the set $W=\bigcup_{i \in[t]}\left(W_{i}^{A} \cup W_{i}^{B}\right)$ has size at most $s \cdot t \cdot 2 \beta d<2 r \beta^{1 / 2} d<$ $\delta^{2} d / r$.

Now, we decide to which part $X_{u}$ we assign $Z_{i}^{C}$. For this, we partition the set [t] into $I_{1}, \ldots, I_{s}, J_{1}, \ldots, J_{q}$ as in the following claim. If $i \in I_{\ell}$, then we will later assign the vertices in $Z_{i}^{A}$ and $Z_{i}^{B}$ to $X_{u_{2 \ell-1}}$ and $X_{u_{2 \ell}}$, respectively. If $i \in J_{\ell^{\prime}}$, then we will later assign the vertices in $Z_{i}^{A}$ and $Z_{i}^{B}$ to $X_{v_{\ell_{\ell^{\prime}}}}$ and $X_{u_{p_{\ell^{\prime}}}}$, respectively. As we do not know how unbalanced the two sets $Z_{i}^{A}$ and $Z_{i}^{B}$ are for each $i$, we prove the following claim using random assignments.

Claim 5.6. There exists a partition $I_{1}, \ldots, I_{s}, J_{1}, \ldots, J_{q}$ of $[t]$ satisfying the following.

- For each $\ell \in[s]$ and $C \in\{A, B\}$, we have $\left|\bigcup_{i \in I_{\ell}} Z_{i}^{C}\right| \leq \frac{\left(1-\delta^{2}\right) d}{r}$.

- For each $\ell^{\prime} \in[q]$, we have $\left|\bigcup_{i \in J_{\ell^{\prime}}} Z_{i}^{A}\right| \leq \frac{\left(1-\delta^{2}\right) d}{r}$ and $\left|\bigcup_{i \in J_{\ell^{\prime}}} Z_{i}^{B} \cup \bigcup_{i \in I_{p_{\ell^{\prime}}}} Z_{i}^{B}\right| \leq$ $\frac{\left(1-\delta^{2}\right) d}{r}$.

Proof of claim. We add each $\ell \in[t]$ independently to one of $I_{1}, \ldots, I_{s}, J_{1}, \ldots, J_{q}$ uniformly at random. Note that for each set, $\ell$ is in the set with probability $1 /(s+q)=1 / r$. Standard concentration inequalities (e.g., Azuma's inequality) easily show that, with 
positive probability, we can ensure that for each $\ell \in[s]$ and $\ell^{\prime} \in[q]$ we have

$$
\begin{aligned}
\left|\bigcup_{i \in I_{\ell}} Z_{i}^{A}\right|,\left|\bigcup_{i \in I_{\ell}} Z_{i}^{B}\right|,\left|\bigcup_{i \in J_{\ell^{\prime}}} Z_{i}^{A}\right| \leq \frac{|A|}{r}+\beta^{1 / 5} d \leq \frac{(1-\delta) d}{(1+\gamma) r}+\beta^{1 / 5} d \leq \frac{\left(1-\delta^{2}\right) d}{r} \\
\quad\left|\bigcup_{i \in I_{\ell_{\ell^{\prime}}}} Z_{i}^{B} \cup \bigcup_{i \in J_{\ell^{\prime}}} Z_{i}^{B}\right| \leq \frac{2|B|}{r}+\beta^{1 / 5} d \leq \frac{2 \gamma(1-\delta) d}{(1+\gamma) r}+\beta^{1 / 5} d \leq \frac{\left(1-\delta^{2}\right) d}{r} .
\end{aligned}
$$

Here, we used $|H|=(1+\gamma)|A| \leq(1-\delta) d$ and $\beta^{1 / 5}<\delta^{2} /(10 r)$ and $2 \gamma /(1+\gamma) \leq 1$ as $\gamma \leq 1$.

With these sets $I_{1}, \ldots, I_{s}, J_{1}, \ldots, J_{q}$, we can distribute the vertices as planned. Assume we have already distributed vertices in $\bigcup_{i \in[k t]} Y_{i}$ to $X_{1}, \ldots, X_{2 s}$ and $X_{p_{1}}^{\prime}, \ldots, X_{p_{q}}^{\prime}$ in such a way that for some $\ell^{\prime} \in[s]$ every vertex in $Y_{k t}^{B}$ is in $X_{2 \ell^{\prime}}$. If $k=0$, then we assume $\ell^{\prime}=0$.

If $k+1 \in I_{\ell}$, choose $\ell^{*} \in[s]$ such that $\ell^{\prime}+\ell^{*} \in\{\ell, \ell+s\}$. We allocate vertices in $Y_{k t+1}^{A}, Y_{k t+1}^{B}, Y_{k t+2}^{A}, \ldots, Y_{k t+\ell^{*}}^{B}$ to $X_{2 \ell^{\prime}+1}, X_{2 \ell^{\prime}+2}, \ldots, X_{2 \ell-1}, X_{2 \ell}$, respectively, and we allocate the remaining vertices in $W_{k+1}^{A} \cup Z_{k+1}^{A}$ to $X_{2 \ell-1}$ and the remaining vertices in $W_{k+1}^{B} \cup Z_{k+1}^{B}$ to $X_{2 \ell}$.

If $k+1 \in J_{\ell}$ for some $\ell \in[q]$, choose $\ell^{*} \in[s]$ such that $\ell^{\prime}+\ell^{*}=p_{\ell}$. We allocate vertices in $Y_{k t+1}^{A}, Y_{k t+1}^{B}, Y_{k t+2}^{A}, \ldots, Y_{k t+\ell^{*}}^{B}$ to $X_{2 \ell^{\prime}+1}, X_{2 \ell^{\prime}+2}, \ldots, X_{p_{\ell}-1}, X_{p_{\ell^{\prime}}}$ respectively and we allocate the remaining vertices in $W_{k+1}^{A} \cup Z_{k+1}^{A}$ to $X_{p_{\ell}}^{\prime}$ and the remaining vertices in $W_{k+1}^{B} \cup Z_{k+1}^{B}$ to $X_{p_{\ell}}$.

By repeating this for $k=0, \ldots, t$, we distribute all vertices. For each $i \in[s]$ and $j \in[q]$, let $X_{u_{i}}=X_{i}$ and $X_{v_{p_{j}}}=X_{p_{j}}^{\prime}$. Then, by the bandwidth condition, all edges of $H$ meeting $Y_{i}^{A}$ are between $Y_{i}^{A}$ and $Y_{i-1}^{B} \cup Y_{i}^{B}$, so we know that each edge of $H$ is between $X_{u}$ and $X_{V}$ for some $u v \in E\left(R_{0}\right)$. As $|W|<\delta^{2} d / r$, for each $u=u_{2 \ell-1}, v=v_{p_{\ell^{\prime}}} \in V\left(R_{0}\right)$ the above distribution ensures that

$$
\left|X_{u}\right| \leq\left|\bigcup_{i \in I_{\ell}} Z_{i}^{A}\right|+|W| \leq \frac{d}{r} \text { and }\left|X_{V}\right| \leq\left|\bigcup_{i \in J_{\ell^{\prime}}} Z_{i}^{A}\right|+|W| \leq \frac{d}{r} .
$$

Again, as $|W|<\delta^{2} d / r$, for each $u=u_{2 \ell}, u^{\prime}=u_{p_{\ell^{\prime}}} \in V\left(R_{0}\right)$ where $2 \ell \notin\left\{p_{1}, \ldots, p_{q}\right\}$, we have

$$
\left|X_{u}\right| \leq\left|\bigcup_{i \in I_{\ell}} Z_{i}^{B}\right|+|W| \leq \frac{d}{r}, \text { and }\left|X_{u^{\prime}}\right| \leq\left|\bigcup_{i \in I_{\ell_{\ell^{\prime}}}} Z_{i}^{B} \cup \bigcup_{i \in J_{\ell^{\prime}}} Z_{i}^{B}\right|+|W| \leq \frac{d}{r}
$$

This proves the lemma. 


\subsection{Proof of Lemma 4.1}

The last ingredient for the dense case is the following result of Voss and Zuluaga providing a long cycle in 2-connected graphs.

Lemma 5.7. [40] Suppose that a graph $R$ is a 2-connected graph on at least $2 d$ vertices with $\delta(G) \geq d$. If $R$ is a bipartite graph with vertex partition $A \cup B$, then $R$ has an even cycle of length at least $\min \{2|A|, 2|B|, 4 d-4\}$. If $R$ is not a bipartite graph, then it has an odd cycle with length at least $\min \{|R|-1,2 d-1\}$.

Proof of Lemma 4.1. Let $d^{\prime}:=d / n>\eta$. We can choose numbers $r_{1} \in \mathbb{N}$ and $\beta, \varepsilon^{\prime}, \tau>0$ such that

$$
0<1 / d \ll \alpha \ll \beta \ll 1 / r_{1} \ll \varepsilon^{\prime} \ll \tau \ll \eta, \delta, \varepsilon<1 .
$$

We apply Lemma 5.1 to $G$ with $\varepsilon^{\prime}, \tau, d^{\prime}$ playing the roles of $\varepsilon, \tau, d$ to obtain a partition $V_{1}, \ldots, V_{r}$ of $V(G)$ and a corresponding reduced graph $R$ with $V(R)=[r], 1 / \varepsilon^{\prime} \leq$ $r \leq r_{1}$, such that $\frac{\left(1-\varepsilon^{\prime}\right) n}{r} \leq\left|V_{i}\right| \leq \frac{\left(1+\varepsilon^{\prime}\right) n}{r}$ for each $i \in[r], G\left[V_{i}, V_{j}\right]$ is $\left(\varepsilon^{\prime}, \tau^{+}\right)$-regular for each $i j \in E(R)$, and $d(R) \geq\left(d^{\prime}-2 \tau\right) r$.

As $d(R) \geq\left(d^{\prime}-2 \tau\right) r$, we can find a 2-connected subgraph $R^{\prime}$ of $R$ with

$$
d\left(R^{\prime}\right) \geq\left(d^{\prime}-3 \tau\right) r \text { and } \delta\left(R^{\prime}\right) \geq \frac{1}{2}\left(d^{\prime}-3 \tau\right) r
$$

One easy way to see such a graph $R^{\prime}$ exists is to apply Lemma 3.2 to $R$ to obtain $R^{\prime}$ with $\tau, 1 / 4$ playing the roles of $\varepsilon_{1}, \varepsilon_{2}$.

Let $r_{0}:=\left(d^{\prime}-4 \tau\right) r$. Now, we will find a graph $R_{0}$ in $R^{\prime}$, which is either an odd cycle of length at least $r_{0}$ or a $(2 a, b)$-sun with $a+b \geq r_{0}$. This will provides a structure in $G$ suitable for us to use the blow-up lemma.

If $R^{\prime}$ is not a bipartite graph, we let $R_{0}$ be an odd cycle of length at least $\min \left\{\left|R^{\prime}\right|-\right.$ $\left.1,2 \delta\left(R^{\prime}\right)-1\right\} \geq r_{0}$ in $R^{\prime}$, as guaranteed by Lemma 5.7 .

If $R^{\prime}$ is bipartite with vertex bipartition $A \cup B$ and $|A| \leq|B|$, then we let $R_{0}$ be a $(2 a, b)$-sun in $R^{\prime}$ for some $a, b$ with $a+b \geq r_{0}$. We claim that such a sun exists. If $|A| \geq r_{0}$, then Lemma 5.7 yields an even cycle $C$ of length at least $\min \left\{2|A|, 4 \delta\left(R^{\prime}\right)-4\right\} \geq 2 r_{0}$ in $R^{\prime}$. This is a $\left(2 r_{0}, 0\right)$-sun as claimed. 
If $|A|<r_{0}$, then let $p=r_{0}-|A|$. As $|A| \leq|B|$, we have

$$
\left(d^{\prime}-3 \tau\right) r \leq d\left(R^{\prime}\right)=\frac{2 e\left(R^{\prime}\right)}{\left|R^{\prime}\right|} \leq \frac{2|A||B|}{|A|+|B|} \leq \min \{2|A|,|B|\}
$$

Thus, we have $p \leq \frac{r_{0}}{2}=\frac{1}{2}\left(d^{\prime}-4 \tau\right) r$ and $r_{0} \leq\left(d^{\prime}-3 \tau\right) r \leq|B|$. Now, as $|A|=r_{0}-p$, we use Lemma 5.7 to find an even cycle $C$ of length at least $\min \left\{2|A|, 4 \delta\left(R^{\prime}\right)-4\right\} \geq 2|A|$ in $R^{\prime}$, which contains all vertices in $A$. Then $|B \backslash V(C)|=|B|-|A|>p$. As each vertex in $B \backslash V(C)$ has at least $\delta\left(R^{\prime}\right) \geq \frac{1}{2}\left(d^{\prime}-3 \tau\right) r>p$ neighbours in $A$, we can find a matching of size at least $\min \{|B \backslash V(C)|, p\} \geq p$ in $R^{\prime}[A, B \backslash V(C)]$. This matching together with the cycle $C$ forms a $(2|A|, p)$-sun with $|A|+p=r_{0}$ as claimed.

Now, using Lemma 5.4 or Lemma 5.5 with $\varepsilon^{\prime}, R_{0},(1-\varepsilon / 2) d$ playing the roles of $\delta, R_{0}, d$, respectively, we can partition $V(H)$ into $\left\{X_{u}: u \in R_{0}\right\}$ where for each $i \in[p]$ we have

$$
\left|X_{i}\right| \leq \frac{(1-\varepsilon / 2) d}{r_{0}} \leq(1-\varepsilon / 3) \frac{n}{r} \leq\left(1-\varepsilon^{\prime}\right)\left|V_{i}\right|
$$

and every edge of $H$ is between $X_{u}$ and $X_{v}$ for some $u v \in E\left(R_{0}\right)$. Hence, 1-3 in Theorem 5.2 are all satisfied with $\varepsilon^{\prime}, \tau, R_{0}$ playing the roles of $\varepsilon, \tau, R_{0}$, respectively, and we conclude that $G$ contains $H$ as a subgraph. This proves the lemma.

\section{Subdivisions in Robust Expanders with Medium Density}

In this section, our goal is to prove Lemma 4.2, which finds an $H$-subdivision in a robust expander with medium density.

We first prove the following lemma, which bounds the number of high degree vertices in our expanders.

Lemma 6.1. Let $0<1 / d \ll \varepsilon_{1}, \varepsilon_{2} \ll \varepsilon, 1 / \Delta<1$, let $H$ be a graph with at most $(1-\varepsilon) d$ vertices satisfying $\Delta(H) \leq \Delta$, and let $G$ be an $n$-vertex graph. Define

$$
m:=\frac{2}{\varepsilon_{1}} \log ^{3}\left(\frac{15 n}{\varepsilon_{2} d}\right), \quad \text { and } \quad L:=\left\{v \in V(G): d_{G}(v) \geq d m^{10}\right\}
$$

Suppose $G$ is an $\left(\varepsilon_{1}, \varepsilon_{2} d\right)$-robust-expander. If $|L| \geq d$, then $G$ contains $H$ as a subdivision.

Proof. Let $V(H)=\left\{x_{1}, \ldots, x_{h}\right\}$ with $h \leq(1-\varepsilon) d$ and let $x_{a_{1}} x_{b_{1}}, \ldots, x_{a_{h^{\prime}}} x_{b_{h^{\prime}}}$ be an arbitrary enumeration of $E(H)$ with $h^{\prime}=e(H) \leq \Delta(1-\varepsilon) d$. 
Take a set $Z=\left\{v_{1}, \ldots, v_{h}\right\}$ of $h$ distinct vertices in $L$. Then, for each $i \in[h]$, the set $X_{i}:=N\left(v_{i}\right)$ has size at least $d m^{10}$. Assume that we have pairwise internally disjoint paths $P_{1}, \ldots, P_{\ell}$ with $0 \leq \ell<h^{\prime}$ where $P_{j}$ is a path between $v_{a_{j}}$ and $v_{b_{j}}$ of length at most $2 m$.

Let $W_{\ell}=\bigcup_{j \in[\ell]} \operatorname{lnt}\left(P_{j}\right)$ be the union of the interior vertices of the paths. As $\left|W_{\ell}\right|+|Z| \leq h^{\prime} \cdot 2 m+h \leq 4 \Delta d m \leq \rho\left(d m^{10}\right) \cdot d m^{10} / 4$, we can apply Lemma 3.3 to get an $X_{a_{\ell+1}}, X_{b_{\ell+1}}$-path $P$ avoiding $W_{\ell} \cup Z$ of length at most $m$. Extending $P$, we obtain a $v_{a_{\ell+1}}, v_{b_{\ell+1}}$-path $P_{\ell+1}$ of length at most $m+2 \leq 2 m$. By repeating this for $\ell=0,1, \ldots, h^{\prime}-1$ in order, we obtain $\bigcup_{j \in\left[h^{\prime}\right]} P_{j}$, which is an $H$-subdivision, in $G$.

Proof of Lemma 4.2. As $\frac{d}{n} \leq \eta \ll \varepsilon_{1}, \varepsilon_{2}$, we have that $m^{100}=\left(\frac{2}{\varepsilon_{1}} \log ^{3}\left(\frac{15 n}{\varepsilon_{2} d}\right)\right)^{100}<\frac{n}{d}$. Hence

$$
d m^{100}<n
$$

To derive a contradiction, we assume that $G$ does not contain an $H$-subdivision. Then by Lemma 6.1, we have

$$
|L|<d
$$

For a contradiction, we will find an $H$-subdivision in $G-L$. For this purpose, we need not only that $L$ is small, but also that the graph $G-L$ is still relatively dense to ensure an $H$-subdivision. We claim that

$$
d(G-L) \geq \frac{d}{m^{2}}
$$

This follows essentially from the robust expansion property of $G$. Indeed, otherwise a random vertex set $X$ of size $d m^{2}$ chosen uniformly at random from $G-L$ has expected degree sum $\mathbb{E}\left[\sum_{X \in X} d_{G-L}(x)\right] \leq \frac{d}{m^{2}} \cdot|X|$. Hence, there exists a set $X \subseteq$ $V(G-L)$ of size $d m^{2}$ with $\sum_{X \in X} d_{G-L}(x) \leq \frac{d}{m^{2}} \cdot|X|$. Then, $F=\partial_{G-L}(X)$ has at most $\frac{d}{m^{2}}|X| \leq d(G) \rho(|X|)|X|$ edges as $d(G) \geq \varepsilon^{2} d$, and $\rho\left(d m^{2}\right) \geq \rho(n)>\frac{1}{m}>\frac{1}{\varepsilon^{2} m^{2}}$ due to $\varepsilon_{1} \ll \varepsilon$. Note that, by definition of $F$, once we delete the edges of $F$ from $G$, the external neighbourhood of $X$ lies entirely in $L$, that is, $N_{G \backslash F}(X) \subseteq L$. However, this implies

$$
\left|N_{G \backslash F}(X)\right| \leq|L| \stackrel{(4)}{<} d \leq \rho\left(d m^{2}\right) \cdot d m^{2}=\rho(|X|) \cdot|X|,
$$

contradicting that $G$ is an $\left(\varepsilon_{1}, \varepsilon_{2} d\right)$-robust-expander. Hence, we have (5). 
Now that $G-L$ is still relatively dense and no vertex in $G-L$ has too large a degree, as $\Delta(G-L) \leq d m^{10}$, we can find many webs with disjoint interiors in $G-L$.

Claim 6.2. The graph $G-L$ contains $\left(m^{2}, m^{10}, d / m^{3}, 4 m\right)$-webs $W_{1}, \ldots, W_{2 d}$ where the interiors of the webs are pairwise disjoint.

To find such webs, we follow the strategy of [14, Lemma 5.7]. We include the proof in the online appendix [12].

Let $W_{1}, \ldots, W_{2 d}$ be the $\left(m^{2}, m^{10}, d / m^{3}, 4 m\right)$-webs guaranteed by the claim. For each $i \in[2 d]$, let $W_{i}$ be the core vertex of the web $W_{i}$ and let $C=\bigcup_{i \in[2 d]} \operatorname{Ctr}\left(W_{i}\right) \cup L$, and so

$$
\left|\operatorname{Ext}\left(W_{i}\right)\right|=d m^{9} \quad \text { and } \quad|C| \leq 2 d\left(m^{2} \cdot 4 m+1\right)+d \leq 10 d m^{3} .
$$

Before moving on, we set up some notation. For a path $Q$ with endvertices $a \in$ $\operatorname{Ext}\left(W_{i}\right)$ and $b \in \operatorname{Ext}\left(W_{j}\right)$, for some distinct $i, j \in[2 d]$ such that $Q \cap\left(\operatorname{Int}\left(W_{i}\right) \cup \operatorname{Int}\left(W_{j}\right)\right)=\varnothing$, we let $Q^{*}=Q \cup P\left(W_{i}, a\right) \cup P\left(W_{j}, b\right)$ be the $w_{i}, W_{j}$-path extending $Q$ in $W_{i} \cup W_{j}$.

For a given set $Z$, we say a web $W$ is $Z$-good if $|\operatorname{lnt}(W) \cap Z| \leq m^{12} / 2$. We define $\left(X, I, I^{\prime}, \mathcal{A}, \mathcal{Q}, f\right)$ be a good path system if the following hold.

A1 $X \subseteq V(H)$ and $f: X \rightarrow[2 d]$ is an injective map with $f(X)=I$.

A2 For each $x \in X$, writing $y_{1}, \ldots, y_{s}$ for the neighbours of $x$ in $H[X], \mathcal{A}$ contains distinct vertices $a_{f(x), f\left(y_{i}\right)} \in \operatorname{Ext}\left(W_{f(x)}\right)$ for $i \in[s]$.

A3 $\mathcal{Q}$ is a collection of paths $O_{i j}$ indexed by unordered pairs $\left(\begin{array}{c}i j \epsilon \\ {[2 d] 2}\end{array}\right)$, such that for each edge $x y \in E(H[X]) \mathcal{Q}$ contains a path $Q_{f(x) f(y)}$ of length at most $m$ with endpoints $a_{f(x), f(y)}$ and $a_{f(y), f(x)}$, and furthermore $Q_{f(x) f(y)} \cap$ $\left(\operatorname{lnt}\left(W_{f(x)}\right) \cup \operatorname{Int}\left(W_{f(y)}\right)\right)=\varnothing$.

A4 For each $x \in X$, writing $y_{1}, \ldots, y_{S}$ for the neighbours of $x$ in $H[X]$, the paths

$$
P\left(W_{f(x)}, a_{f(x), f\left(y_{1}\right)}\right), \ldots, P\left(W_{f(x)}, a_{f(x), f\left(y_{s}\right)}\right)
$$

are pairwise disjoint except at $w_{f(x)}$.

A5 $\left\{O_{i j}^{*}-C: O_{i j} \in \mathcal{Q}, i j \in[2 d] 2\right\}$ is a collection of pairwise disjoint paths in $G-C$.

A6 $I^{\prime}=\left\{i^{\prime} \in[2 d]: W_{i^{\prime}}\right.$ is $\left.\operatorname{not} V(\mathcal{Q})-\operatorname{good}\right\} \subseteq[2 d] \backslash I$.

We shall show that there is a good path system with $X=V(H)$, which would finish the proof as by A1-A5, $\bigcup_{e \in E(H)} Q_{e}^{*}$ is an $H$-subdivision. 
We proceed as follows (see Figure 4 for an example).

Step 0. Fix an arbitrary ordering $\sigma$ on $V(H)$, say the first vertex is $x_{1}$. Let $X_{1}=\left\{x_{1}\right\}$, $f\left(x_{1}\right)=1, I_{1}:=\{1\}, I_{1}^{\prime}:=\varnothing, \mathcal{A}_{1}=\varnothing$ and $\mathcal{Q}_{1}=\varnothing$. Then, $\left(X_{1}, I_{1}, I_{1}^{\prime}, \mathcal{A}_{1}, \mathcal{Q}_{1}, f\right)$ is a good path system. Proceed to Step 1.

Step $i, i \geq 1$. Stop if either $X_{i}=V(H)$, or $I_{i} \cup I_{i}^{\prime}=[2 d]$.

- Add a new vertex.

Let $x$ be the first vertex in $\sigma$ on $V(H) \backslash X_{i}$. Choose an unused $V\left(\mathcal{Q}_{i}\right)$-good web $W_{i^{*}}$ with $i^{*} \in[2 d] \backslash\left(I_{i} \cup I_{i}^{\prime}\right)$ and let $f(x)=i^{*}$.

Find vertices $a_{f(x), f\left(y_{1}\right)}, \ldots, a_{f(x), f\left(y_{s}\right)}$ and $a_{f\left(y_{1}\right), f(x)}, \ldots, a_{f\left(y_{s}\right), f(x)}$, together with paths $Q_{f(x) f\left(y_{1}\right)}, \ldots, Q_{f(x) f\left(y_{s}\right)}$, satisfying A2-A5 with respect to $X_{i} \cup$ $\{x\}$, and add these vertices to $\mathcal{A}_{i}$ and paths to $\mathcal{Q}_{i}$ to obtain $\mathcal{A}_{i+1}$ and $\mathcal{Q}_{i+1}$.

- Update bad webs.

- Let $I_{i+1}^{\prime}=\left\{i^{\prime} \in[2 d]: W_{i^{\prime}}\right.$ is $\operatorname{not} V\left(\mathcal{Q}_{i+1}\right)$-good $\}$.

- Set $I_{i+1}=I_{i} \cup\left\{i^{*}\right\} \backslash I_{i+1}^{\prime}$ and $X_{i+1}=f^{-1}\left(I_{i+1}\right)$.

- Replace $f$ with its restriction $\left.f\right|_{X_{i+1}}$ on $X_{i+1}$.

Proceed to Step $(i+1)$ with the new good path system $\left(X_{i+1}, I_{i+1}, I_{i+1}^{\prime}, \mathcal{A}_{i+1}, \mathcal{Q}_{i+1}, f\right)$.

The above process must terminate in at most $2 d$ steps, since at each step $I_{i+1} \cup I_{i+1}^{\prime}$ is a strict superset of $I_{i} \cup I_{i}^{\prime}$. Let $\left(X, I, I^{\prime}, \mathcal{A}, \mathcal{Q}, f\right)$ be the final good path system obtained. Note that the sequence $\left|X_{1}\right|,\left|X_{2}\right|, \ldots$ might not be an increasing sequence, as we may delete some elements when updating the list of bad webs in each step. However, we will show that eventually $X=V(H)$ as desired.

First, we claim that $\left|I^{\prime}\right| \leq d / m$. Note that $\mathcal{Q}$ might contain some paths which connect $W_{i}$ with $i \in I^{\prime}$. However, as at most $\Delta(H) \leq \Delta$ paths are added at each step,

$$
|V(\mathcal{Q})| \leq 2 d \cdot \Delta(m+1) \leq d m^{2}
$$

Recall that $\left\{\operatorname{Int}\left(W_{j}\right): j \in[2 d]\right\}$ are pairwise disjoint and so, by definition of $I^{\prime},\left|I^{\prime}\right| \cdot m^{12} / 2 \leq$ $|V(\mathcal{Q})|$. Thus, $\left|I^{\prime}\right| \leq \frac{|V(\mathcal{Q})|}{m^{12} / 2}<d / m$ as claimed.

Now since $\left|I_{i} \cup I_{i}^{\prime}\right| \leq|H|+\left|I^{\prime}\right|<2 d$, the process must terminate with $X=V(H)$. To finish the proof, it only remains to show that all connections in each step, that is, vertices in $\mathcal{A}_{i+1} \backslash \mathcal{A}_{i}$ and paths in $\mathcal{Q}_{i+1} \backslash \mathcal{Q}_{i}$, can indeed be constructed to keep the process running.

Let $x, i^{*}=f(x)$ and $\left\{y_{1}, \ldots, y_{s}\right\}=N_{H}(x) \cap\left(X_{i} \cup\{x\}\right)$ be as in Step $i$, for some $i \geq 1$. Consider now $j \in I_{i} \cup\left\{i^{*}\right\}=f\left(X_{i} \cup\{x\}\right)$. Note that as $\left(X_{i}, I_{i}, I_{i}^{\prime}, \mathcal{A}_{i}, \mathcal{Q}_{i}, f\right)$ is a good path system at the beginning of this step, by $\mathbf{A 6}, W_{j}$ is $V\left(\mathcal{Q}_{i}\right)$-good. Also, as $V\left(\mathcal{Q}_{i}\right)$ is disjoint from $C$ 


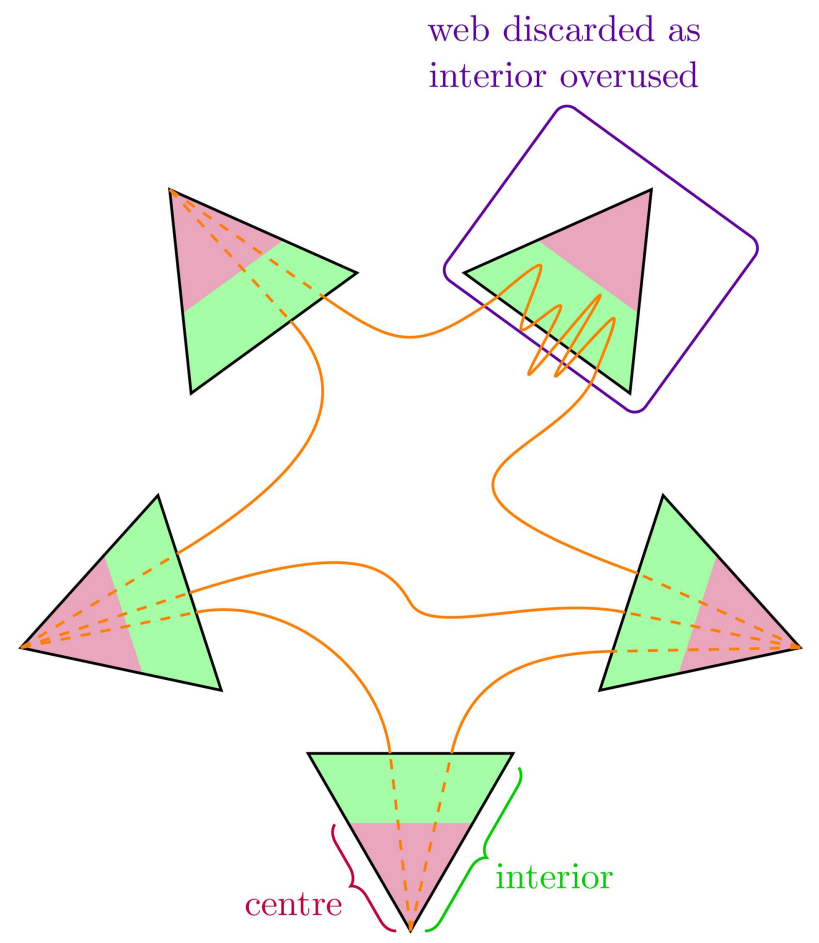

Fig. 4. An example for $H=K_{1,1,2}$ (the diamond graph).

by $\mathbf{A} 3$ and $\mathbf{A} 5$, at most $\Delta(H) \leq \Delta$ paths in $\operatorname{Ctr}\left(W_{j}\right)$ are involved in previous connections $\mathcal{Q}_{i}^{*}:=\left\{Q^{*}: Q \in \mathcal{Q}_{i}\right\}$. Thus, there are at least $\left(m^{2}-\Delta\right) m^{10}-m^{12} / 2 \geq m^{12} / 4$ available paths in $\operatorname{Int}\left(W_{j}\right) \backslash \operatorname{Ctr}\left(W_{j}\right)$ (and their corresponding paths in $\left.\operatorname{Ctr}\left(W_{j}\right)\right)$ disjoint from $V\left(\mathcal{Q}_{i}^{*}\right)$; let $A_{j} \subseteq \operatorname{Ext}\left(W_{j}\right)$ be the union of the leaves of the stars corresponding to these available paths. Then $\left|A_{j}\right| \geq a:=d m^{9} / 4$.

Now, for each $j \in[s]$, since

$$
\left|C \cup \operatorname{Int}\left(W_{i^{*}}\right) \cup \operatorname{Int}\left(W_{f\left(y_{j}\right)}\right) \cup V\left(\mathcal{Q}_{i}^{*}\right)\right| \leq 10 d m^{3}+20 m \cdot m^{12}+30 m|\mathcal{Q}| \leq d m^{4} \leq \rho(a) a / 4,
$$

using Lemma 3.3, we can find the desired path $Q_{i^{*} f\left(y_{j}\right)}$, with length at most $m$, connecting $A_{i^{*}}$ and $A_{f\left(y_{j}\right)}$ while avoiding $C \cup \operatorname{Int}\left(W_{i^{*}}\right) \cup \operatorname{lnt}\left(W_{f\left(y_{j}\right)}\right) \cup V\left(\mathcal{Q}_{i}^{*}\right)$, and take $a_{i^{*}, f\left(y_{j}\right)}$ and $a_{f\left(y_{j}\right), i^{*}}$ to be its endpoints.

This finishes the proof of Lemma 4.2. 


\section{Subdivisions in Sparse Robust Expanders}

In this section, we prove Lemma 4.3. First, we show in Section 7.1 that we cannot have too many edges sticking out of a small set of vertices, for otherwise we can obtain $H$ even as a subgraph in $G$. In particular, $G-L$ is still dense (Claim 7.1), and consequently it must contain a subexpander $F$ with $d(F)=\Omega(d)$.

Note that this subexpander could be a lot smaller and hence we have no control on its density. Suppose for a moment that $F$ is sparse, and additionally $F$ is large, then it inherits the 'bounded' maximum degree from $G-L$. This case is handled in Section 7.2. For such an 'almost regular' sparse expander, we can work entirely within it to find an $H$-subdivision (Lemma 7.3).

Now, if $F$ has medium edge density, then we can invoke Lemma 4.2 on $F$ and we are done. Therefore, $F$ must either be sparse and small, or very dense. In both cases, $F$ is small in order. To this end, we may assume that all subexpanders in $G-L$ are small. In Section 7.3, we shall iteratively pull out many small expanders in $G-L$ that are pairwise far apart (C1-C4), most of which expand well inside $G-L$ (Claim 7.4).

We then in Section 7.4 link these nicely expanding small expanders to construct nakjis (Claim 7.5). The strategy here is to extract nakjis iteratively. Every time, we step far away in $G-L$ from previously built nakjis and expand an appropriate collection of expanders to find the next one.

Finally, the finishing blow is delivered in Section 7.5, in which we anchor on the nakjis and connect them to build an $H$-subdivision.

\section{1 $G-L$ still dense}

As $d<m^{100}=\left(\frac{2}{\varepsilon_{1}} \log ^{3}\left(\frac{15 n}{\varepsilon_{2} d}\right)\right)^{100}$ and $1 / d \ll 1 / \Delta$, we have $n>10 \Delta d^{\Delta}$. Suppose to the contrary that $G$ does not have any $H$-subdivision. Then by Lemma $6.1,|L|<d$.

We first establish the following claim, stating that the density does not drop much upon removing a small set of vertices. The idea is that if lots of edges are incident to a small set, then we will see a dense and skewed bipartite subgraph, which contains a copy of $H$.

Claim 7.1. For any set $U \subseteq V(G-L)$ of size at most $n / m^{200}$, we have $d(G-L-U) \geq \varepsilon d / 6$.

Proof of claim. Let

$$
Z:=\left\{V \in V(G-L):\left|N_{G}(V, L)\right|>(1-\varepsilon) d / 2\right\}
$$


Suppose that $|Z|>\Delta d^{\Delta}$. For a given function $f: Z \rightarrow\left(\begin{array}{l}L \\ \Delta\end{array}\right)$, we let

$$
t(f):=\sum_{Y \in\left(\begin{array}{c}
L \\
\Delta
\end{array}\right)} \min \left\{\left|f^{-1}(Y)\right|, \Delta\right\} .
$$

Let $f: Z \rightarrow\left(\begin{array}{l}L \\ \Delta\end{array}\right)$ be a function with maximum $t(f)$ among all functions satisfying $f(v) \subseteq$ $N_{G}(v, L)$ for every $v \in Z$. As $\left|N_{G}(v, L)\right| \geq \Delta$, such a function $f$ must exist. However, as $|Z|>\Delta d^{\Delta}>\Delta\left(\begin{array}{c}|L| \\ \Delta\end{array}\right)$, there exist $\Delta+1$ vertices $u_{1}, \ldots, u_{\Delta+1} \in Z$ with $f\left(u_{1}\right)=\cdots=f\left(u_{\Delta+1}\right)$. Let $X=N_{G}\left(u_{1}, L\right)$. If any set $Y \in\left(\begin{array}{c}X \\ \Delta\end{array}\right)$ satisfies $\left|f^{-1}(Y)\right|<\Delta$, then we can redefine $f\left(u_{1}\right)$ to be $Y$ to increase $t(f)$. Hence, by the maximality of $f$, for every set $Y \in\left(\begin{array}{l}X \\ \Delta\end{array}\right)$, there are $\Delta$ distinct vertices $v_{Y}^{1}, \ldots, v_{Y}^{\Delta} \in Z$ such that $Y \subseteq N\left(v_{Y}^{i}, L\right)$ for each $i \in[\Delta]$ and $v_{Y}^{i} \neq v_{Y^{\prime}}^{j}$ for all $Y \neq Y^{\prime} \in\left(\begin{array}{l}X \\ \Delta\end{array}\right)$ and $i, j \in[\Delta]$. It is easy to see that $X$ together with the vertices $\left\{v_{Y}^{i}: Y \in\left(\begin{array}{c}X \\ \Delta\end{array}\right), i \in[\Delta]\right\}$ induces a graph containing any $(1-\varepsilon) d$-vertex bipartite graph with maximum degree at most $\Delta$. Hence, $G$ contains $H$ as a subgraph, a contradiction. Thus, $|Z| \leq \Delta d^{\Delta}$.

As $n \geq 10 \Delta d^{\Delta}$ and $|Z \cup L|<2 \Delta d^{\Delta}$, at least $4 n / 5$ vertices in $V(G)-L-Z$ have $\delta(G)-\frac{1}{2}(1-\varepsilon) d \geq \varepsilon d / 4$ neighbours in $G-L$. Hence, $e(G-L) \geq \frac{1}{2}(4 n / 5) \cdot(\varepsilon d / 4) \geq \varepsilon d n / 10$. Then, $G-L-U$ has at least $e(G-L)-\Delta(G-L)|U| \geq \varepsilon d n / 10-d m^{10} \cdot\left(n / m^{200}\right) \geq \varepsilon d n / 12$ edges.

From this point on, we will work with $G^{\prime}:=G-L$. Recall that

$$
\Delta\left(G^{\prime}\right) \leq d m^{10} \leq m^{110}
$$

\section{2 'Bounded' degree sparse expander}

As outlined at the start, since $G-L$ is still dense, it contains a subexpander $F$ at our disposal. We first take care of the case when this subexpander is sparse and 'almost regular'.

We will use the following proposition to take many vertices that are pairwise far apart ([21, Proposition 5.3] taking $s=2000)$.

Proposition 7.2. Suppose that $F$ is an $n$-vertex graph with $\Delta(F) \leq \log ^{50000} n$, and $n$ sufficiently large. Then there is a set of at least $n^{1 / 5}$ vertices pairwise having distance at least $\frac{\log n}{100000 \log \log n}$. 
Lemma 7.3. Let $0 \ll 1 / d \ll \varepsilon_{1}, \varepsilon_{2} \ll \varepsilon, 1 / \Delta<1$ and let $H$ be a graph with at most $d$ vertices and $\Delta(H) \leq \Delta$. Suppose $F$ is an $n$-vertex $\left(\varepsilon_{1}, \varepsilon_{2} d\right)$-robust-expander with $\delta(F) \geq$ $\varepsilon^{2} d$. If $\Delta(F) \leq \log ^{30000} n$, then $F$ contains an $H$-subdivision.

Proof. Let

$$
r:=(\log \log n)^{5} \quad \text { and } \quad r^{\prime}=\sqrt{\log n}
$$

As $d \leq \delta(F) / \varepsilon^{2} \leq \Delta(F) / \varepsilon^{2} \leq \log ^{30001} n$, Proposition 7.2 implies that we can find vertices $v_{1}, \ldots, v_{h}$, where $h=|H| \leq d$, such that the distance between any two of them is at least $2 r+2 r^{\prime}$. Let $x_{1}, \ldots, x_{h}$ be the vertices of $H$ and $e_{1}=x_{a_{1}} x_{b_{1}}, \ldots, e_{h^{\prime}}=x_{a_{h^{\prime}}} x_{b_{h^{\prime}}}$ be the edges of $H$ where $h^{\prime}=e(H) \leq \Delta d / 2$.

Suppose that we have $Q_{1}, \ldots, Q_{\ell}$ for some $0 \leq \ell<h^{\prime}$ such that:

B1 for each $i \in[\ell], O_{i}$ is a $v_{a_{i}}, v_{b_{i}}$-path with length at most $2 \log ^{4} n$;

B2 for distinct $i, j \in[\ell]$, the paths $Q_{i}$ and $Q_{j}$ are internally vertex disjoint;

B3 for each $i \in[h]$, for the edges $\left\{e_{k_{1}}, \ldots, e_{k_{s}}\right\}=\left\{e_{k_{j}}: k_{j} \in[\ell], x_{i} \in e_{k_{j}}\right\}$ with $k_{1}<\cdots<k_{s^{\prime}}$ the paths $Q_{k_{1}}, \ldots, Q_{k_{s}}$ form consecutive shortest paths from $N\left(v_{i}\right)$ within $B^{r}\left(v_{i}\right) \backslash\left\{v_{i}\right\}$; and

B4 for any $i \in[h]$ and $j \in[\ell]$ with $x_{i} \notin e_{j}, B^{r}\left(V_{i}\right)$ and $V\left(O_{j}\right)$ are disjoint.

Let

$$
W_{1}=\bigcup_{i \in[\ell]} \operatorname{lnt}\left(Q_{i}\right), \quad W_{2}:=\bigcup_{i \in[h]: x_{i} \notin e_{\ell+1}} B^{r}\left(v_{i}\right) \quad \text { and } \quad W=W_{1} \cup W_{2} \text {. }
$$

Note that $v_{1}, \ldots, v_{h}$ are pairwise a distance at least $2 r+2 r^{\prime}$ apart, so by $\mathbf{B} 4$ we have

$$
\left|B_{F-W}^{r}\left(V_{k_{\ell+1}}\right)\right|=\left|B_{F-W_{1}}^{r}\left(v_{k_{\ell+1}}\right)\right|=\left|B_{F-W_{1}}^{r-1}\left(B_{F-W_{1}}^{1}\left(v_{k_{\ell+1}}\right)\right)\right|
$$

for each $k \in\{a, b\}$. Note that $\left|B_{F-W_{1}}^{1}\left(v_{k_{\ell+1}}\right)\right| \geq \varepsilon^{2} d-\Delta \geq \varepsilon^{2} d / 2$ by B3 and B4. By B3, we can apply Proposition 3.5 with $B_{F-W_{1}}^{1}\left(v_{k_{\ell+1}}\right), W_{1}, \varnothing, \Delta$ playing the roles of $X, P, Y, q$, and then for each $k \in\{a, b\}$ we have

$$
\left|B_{F-W}^{r}\left(v_{k_{\ell+1}}\right)\right|=\left|B_{F-W_{1}}^{r}\left(v_{k_{\ell+1}}\right)\right| \geq \exp \left((r-1)^{1 / 4}\right) \geq d \log ^{8} n,
$$

where the last inequality follows from $d \leq \log ^{30001} n$. This implies that

$$
\left|W_{1}\right| \leq h^{\prime} \cdot 2 \log ^{4} n \leq \Delta d \log ^{4} n<\frac{1}{4} \rho\left(\left|B_{F-W}^{r}\left(v_{k_{\ell+1}}\right)\right|\right) \cdot\left|B_{F-W}^{r}\left(v_{k_{\ell+1}}\right)\right| \text {. }
$$


Hence, by applying Proposition 3.5, now with $B_{F-W}^{r}\left(v_{k_{\ell+1}}\right), \varnothing, W_{1}$ playing the roles of $X, P, Y$ for each $k \in\{a, b\}$, we similarly have

$$
\left|B_{F-W}^{r+r^{\prime}}\left(v_{k_{\ell+1}}\right)\right|=\left|B_{F-W_{1}}^{r+r^{\prime}}\left(v_{k_{\ell+1}}\right)\right| \geq \exp \left(\left(r^{\prime}\right)^{1 / 4}\right) \geq \exp (\sqrt[9]{\log n}) .
$$

As $\Delta(F) \leq \log ^{30000} n$ and $d \leq \log ^{30001} n$, we then have

$$
|W| \leq\left|W_{1}\right|+\left|W_{2}\right| \leq \Delta d \log ^{4} n+d \cdot 2\left(\log ^{30000} n\right)^{r}<\frac{1}{4} \rho(\exp (\sqrt[9]{\log n})) \exp (\sqrt[9]{\log n}) .
$$

Therefore, by Lemma 3.3 , there is a path in $F-W$ of length at most $\log ^{4} n$ between $B_{F-W}^{r+r^{\prime}}\left(V_{a_{\ell+1}}\right)$ and $B_{F-W}^{r+r^{\prime}}\left(V_{b_{\ell+1}}\right)$. So, we can take $Q_{\ell+1}$ to be a shortest path between $V_{a_{\ell+1}}$ and $v_{b_{\ell+1}}$ in $F-W$, which has length at most $\log ^{4} n+2 r+2 r^{\prime} \leq 2 \log ^{4} n$. Hence, $Q_{1}, \ldots, Q_{\ell+1}$ satisfy B1-B4. Repeating this for $\ell=0,1, \ldots, h^{\prime}-1$, then the union of all paths $\bigcup_{i \in\left[h^{\prime}\right]} Q_{i}$ is an $H$-subdivision.

\subsection{Many small subexpanders}

With Lemma 7.3 at hand, we can now proceed to show that all subexpanders in $G^{\prime}=G-L$ must be small and that we can find many of them pairwise far apart.

Let $\mathcal{F}$ be a maximal collection of subgraphs of $G^{\prime}$ satisfying the following.

C1 For each $F \in \mathcal{F}, F$ is an $\left(\varepsilon_{1}, \varepsilon_{2} d\right)$-expander with $d(F) \geq \varepsilon d / 10$ and $\delta(F) \geq$ $\varepsilon d / 20$ and $F$ is $\varepsilon^{2} v d$-connected.

C2 For distinct $F, F^{\prime} \in \mathcal{F}$, we have $B_{G^{\prime}}^{\sqrt{\log n}}(V(F)) \cap B_{G^{\prime}}^{\sqrt{\log n}}\left(V\left(F^{\prime}\right)\right)=\varnothing$.

For each $F \in \mathcal{F}$, let

$$
n_{F}=|F|, \quad m_{F}=\frac{2}{\varepsilon_{1}} \log ^{3}\left(\frac{15 n_{F}}{\varepsilon_{2} d}\right) \quad \text { and } \quad U=\bigcup_{F \in \mathcal{F}} B_{G^{\prime}}^{2 \sqrt{\log n}}(V(F)) .
$$

If some $F \in \mathcal{F}$ satisfies $m_{F}^{100}<d(F)<\sqrt{n_{F}}$, then $F$ satisfies the conditions on Lemma 4.2. Hence, Lemma 4.2 yields an $H$-subdivision in $F$, a contradiction. Thus, either

$$
n_{F} \leq d(F)^{2} \leq \Delta\left(G^{\prime}\right)^{2} \stackrel{(6)}{\leq} m^{220} \quad \text { or } \quad d(F) \leq m_{F}^{100}
$$

If the latter case holds, we claim that $n_{F} \leq \exp (\sqrt[50]{\log n})$. Indeed, if $n_{F}>\exp (\sqrt[50]{\log n})$ holds, then we have

$$
\Delta(F) \leq \Delta\left(G^{\prime}\right) \stackrel{(6)}{\leq} m^{110} \leq \log ^{30000} n_{F}
$$


So, we can apply Lemma 7.3 on $F$ to get an $H$-subdivision, a contradiction. Thus, we have

C3 for each $F \in \mathcal{F}, n_{F} \leq \max \left\{m^{220}, \exp (\sqrt[50]{\log n})\right\}=\exp (\sqrt[50]{\log n})$.

Moreover, if $|\mathcal{F}|<n^{0.99}$, then by (6) this implies that

$$
|U| \leq n^{0.99} \cdot \exp (\sqrt[50]{\log n}) \cdot 2 \Delta\left(G^{\prime}\right)^{2 \sqrt{\log n}} \leq \frac{n}{m^{200}}
$$

Hence, Claim 7.1 implies that $G^{\prime}-U$ has average degree at least $\varepsilon d / 6$. Thus Lemma 3.2 finds another expander $F$ satisfying $\mathbf{C} 1$ in $G^{\prime}-U$. Then $\mathbf{C 2}$ also holds, contradicting the maximality of $\mathcal{F}$. Hence, we have

$$
\text { C4 }|\mathcal{F}| \geq n^{0.99}
$$

Furthermore, the following claim states that most of the expanders in $\mathcal{F}$ expand well in $G^{\prime}$.

Claim 7.4. There exist at least $|\mathcal{F}|-d \log n$ graphs $F$ in $\mathcal{F}$ such that for each $1 \leq r \leq \log n$,

$$
\left|B_{G^{\prime}}^{r}(V(F))\right| \geq \frac{1}{d} \exp \left(r^{1 / 4}\right)
$$

Proof of claim. Fix a choice of $1 \leq r \leq \log n$ and consider a set $\mathcal{I} \subseteq \mathcal{F}$ with $|\mathcal{I}|=d$ and let $X=\bigcup_{F \in \mathcal{I}} V(F)$. Note that by $\mathbf{C 1},|X| \geq \varepsilon d^{2} / 10$, and so $|L|<d \leq \frac{1}{4} \rho(|X|)|X|$. By Proposition 3.5 with $L$ playing the role of $Y$, we have

$$
\left|B_{G^{\prime}}^{r}(X)\right| \geq \exp \left(r^{1 / 4}\right)
$$

Then by the pigeonhole principal, there exists $F \in \mathcal{I}$ such that

$$
\left|B_{G^{\prime}}^{r}(V(F))\right| \geq \frac{1}{d} \exp \left(r^{1 / 4}\right)
$$

Therefore, whenever there are $d$ members left in $\mathcal{F}$, we can keep picking out one, the $r$-ball around which expands nicely in $G^{\prime}$. Now varying $r$, we see that there are at least $|\mathcal{F}|-d \log n$ graphs $F$ in $\mathcal{F}$ as claimed.

By losing a factor of 2 in size, that is, instead of $C 4,|\mathcal{F}| \geq n^{0.99} / 2$, we may assume now every member in $\mathcal{F}$ expands well in $G^{\prime}$ as in Claim 7.4 


\subsection{Linking subexpanders to nakjis}

We will now connect these far apart nicely expanding subexpanders in $\mathcal{F}$ to obtain $d$ separate $(\Delta, \exp (\sqrt[50]{\log n}), m, 2 \sqrt{\log n})$-nakjis to anchor.

More precisely, take the following collections with maximum possible $p \in \mathbb{N}$.

D1 $\mathcal{C}:=\left\{C_{i, j}: i \in[p], 0 \leq j \leq \Delta\right\}$ is a collection of vertex sets of distinct graphs in $\mathcal{F}$.

D2 For each $(i, j) \in[p] \times[\Delta]$, we have a vertex set $S_{i, j}$ with $C_{i, j} \subseteq S_{i, j} \subseteq B_{G^{\prime}}^{\sqrt[10]{\log n}}\left(C_{i, j}\right)$ with $\left|S_{i, j}\right|=\exp (\sqrt[50]{\log n})$ and $G\left[S_{i, j}\right]$ is connected.

D3 $\left\{P_{i, j}:(i, j) \in[p] \times[\Delta]\right\}$ is a collection of pairwise vertex disjoint paths, each of length at most $10 \mathrm{~m}$, such that $P_{i, j}$ is a $u_{i, j}, v_{i, j}$-path where $u_{i, j} \in C_{i, 0}$ and $v_{i, j} \in S_{i, j}$ and the internal vertices of $P_{i, j}$ are not in $C_{i, 0} \cup S_{i, j}$.

D4 For distinct $(i, j),\left(i^{\prime}, j^{\prime}\right) \in[p] \times[\Delta], P_{i, j}$ is disjoint from $S_{i^{\prime}, j^{\prime}}$ and $\bigcup_{i^{\prime \prime} \in[p] \backslash\{i\}} C_{i^{\prime \prime}, 0}$.

So, by C1 and D1-D4, we see that for each $i \in[p], \bigcup_{0 \leq j \leq \Delta} C_{i, j}$ and $\bigcup_{j \in[\Delta]} P_{i, j}$ form a $(\Delta, \exp (\sqrt[50]{\log n}), m, 2 \sqrt{\log n})$-nakji, in which $C_{i, 0}$ is the head and each $C_{i, j}, j \in[\Delta]$, is a leg.

Claim 7.5. We have $p \geq d$.

Proof of claim. Suppose to the contrary that $p<d$. Let $W$ be the set of vertices involved in $\mathcal{C}$ and all the paths $P_{i, j}$, and $W^{\prime}$ be a $\sqrt{\log n}$-ball in $G^{\prime}$ around $W$. That is,

$$
W=\bigcup_{(i, j) \in[p] \times[\Delta]} V\left(P_{i, j}\right) \cup \bigcup_{C \in \mathcal{C}} V(C), \quad \text { and } \quad W^{\prime}=B_{G^{\prime}}^{\sqrt{\log n}}(W)
$$

Then, by D1-D3 and (6), we see that

$$
|W| \leq(\Delta+1) d \cdot(\exp (\sqrt[50]{\log n})+20 m) \leq \exp (\sqrt[49]{\log n})
$$

and

$$
\left|W^{\prime}\right| \leq \exp (\sqrt[49]{\log n}) \cdot 2 \Delta\left(G^{\prime}\right)^{\sqrt{\log n}} \leq n^{0.1}
$$

We shall find a nakji in $G^{\prime}-W^{\prime}$, which will lead to a contradiction to the maximality of $p$.

As $|\mathcal{F}|-\left|W^{\prime}\right|>n^{0.98}$, we can choose two disjoint collections $\mathcal{F}_{0}, \mathcal{F}^{\prime} \subseteq \mathcal{F}$, containing subexpanders disjoint from $W^{\prime}$, with $\left|\mathcal{F}_{0}\right|=d$ and $\left|\mathcal{F}^{\prime}\right|=n^{0.97}$. Let $X=$ $\bigcup_{F \in \mathcal{F}_{0}} V(F)$, then by $\mathrm{C} 1$, we have $|X| \geq \frac{\varepsilon d^{2}}{10}$. 
Now assume that, for some $0 \leq \ell \leq \Delta d$, we have pairwise vertex disjoint paths $Q_{1}, \ldots, Q_{\ell}$ in $G^{\prime}$ satisfying the following for each $i \in[\ell]$.

E1 $Q_{i}$ is a path of length at most $10 m$ from $X$ to $F_{i}^{\prime} \in \mathcal{F}^{\prime}$.

E2 $Q_{1}, \ldots, Q_{\ell}$ are consecutive shortest paths from $X$ within $B_{G^{\prime}}^{\sqrt{\log n}}(X)$.

E3 $Q_{i}$ is disjoint from $W \cup \bigcup_{j \in[\ell] \backslash\{i\}} B_{G^{\prime}} \sqrt[10]{\log n}\left(V\left(F_{j}^{\prime}\right)\right)$ and it is a shortest path from $V\left(F_{i}^{\prime}\right)$ in $B_{G^{\prime}}^{\sqrt[10]{\log n}}\left(V\left(F_{i}^{\prime}\right)\right)$

Let

$$
Q=\bigcup_{i \in[\ell]} V\left(Q_{i}\right), \quad Q^{\prime}=B{\sqrt[10]{G^{\prime}}}^{\log n}(Q) \quad \text { and } \quad W^{*}=\bigcup_{i \in[\ell]} B B_{G^{\prime}}^{\sqrt[10]{\log n}}\left(V\left(F_{i}^{\prime}\right)\right)
$$

then we have by $\mathbf{C 3}$ and (6) that

$$
\begin{gathered}
|Q| \leq d^{2} m, \quad\left|Q^{\prime}\right| \leq d^{2} m \cdot 2 \Delta\left(G^{\prime}\right) \sqrt[10]{\log n} \leq \exp (\sqrt[9]{\log n}) \quad \text { and } \\
\left|W^{*}\right| \leq \ell \exp (\sqrt[50]{\log n}) \cdot 2 \Delta\left(G^{\prime}\right) \sqrt[10]{\log n} \leq \exp (\sqrt[9]{\log n})
\end{gathered}
$$

Let $\mathcal{F}^{\prime \prime}$ be the graphs in $\mathcal{F}^{\prime} \backslash\left\{F_{1}^{\prime}, \ldots, F_{\ell}^{\prime}\right\}$, which do not intersect with $Q^{\prime}$. Then, letting $U=\bigcup_{F \in \mathcal{F}^{\prime \prime}} V(F)$, we have

$$
|U| \geq\left|\mathcal{F}^{\prime}\right|-\ell-\left|Q^{\prime}\right| \geq n^{0.97}-d^{2}-\exp (\sqrt[6]{\log n}) \geq n^{0.9}
$$

We shall connect $X$ and $U$. First we expand $X$ as follows. As $|X| \geq \varepsilon d^{2} / 10,|L| \leq d$ and $\ell \leq \Delta d \leq \frac{|X|}{\log ^{8}|X|}$, E2 implies that we can apply Proposition 3.5 with $X, Q, L$ playing the roles of $X, P, Y$ to obtain that

$$
\left|B_{G^{\prime}-Q-W-W^{*}}^{\sqrt{\log n}}(X)\right|=\left|B_{G^{\prime}-Q}^{\sqrt{\log n}}(X)\right| \geq \exp (\sqrt[8]{\log n})
$$

where the first equality follows from $X$ being far from $W \cup W^{*}$ owing to $X \cap W^{\prime}=\varnothing$ and C2.

As

$$
\left|L \cup Q \cup W \cup W^{*}\right| \leq 2 \exp (\sqrt[9]{\log n})<\frac{1}{4} \rho(\exp (\sqrt[8]{\log n})) \cdot \exp (\sqrt[8]{\log n})
$$

and $|U| \geq n^{0.9}$, Lemma 3.3 implies that there exists a path of length at most $m$ between the sets $U$ and $B_{G^{\prime}-Q-W-W^{*}}^{\sqrt{\log n}}(X)$ avoiding $L \cup Q \cup W \cup W^{*}$. Let $O_{\ell+1}^{\prime}$ be a shortest such path with endvertices say $v \in B_{G^{\prime}-Q-W-W^{*}}^{\sqrt{\log n}}(X)$ and $u \in U$ (see Figure 5). Let $F_{\ell+1}^{\prime} \in \mathcal{F}^{\prime \prime}$ be 


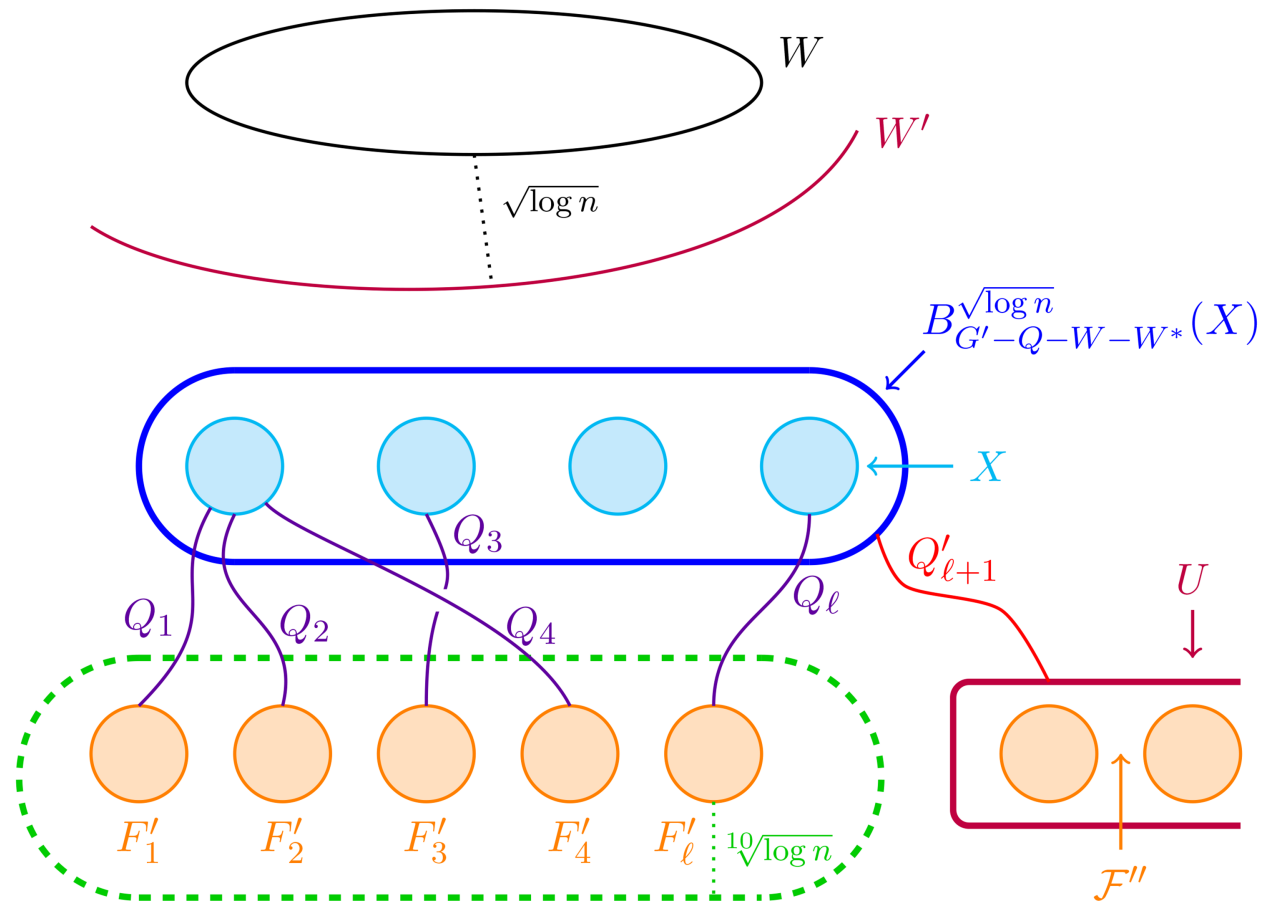

Fig. 5. The proof of Claim 7.5.

the graph containing $u$. Appending a shortest path in $G^{\prime}-Q$ from a vertex of $X$ to $V$ in $B_{G^{\prime}-Q}^{\sqrt{\log n}}(X)$ to the path $Q_{\ell+1}^{\prime}$, let the resulting path be $Q_{\ell+1}$. The choices of $W^{*}, F_{\ell+1}^{\prime}$ and the path $Q_{\ell+1}$ ensure that E1-E3 hold for $Q_{1}, \ldots, Q_{\ell+1}$.

Repeating this for $\ell=0, \ldots, d \Delta$, we obtain paths $Q_{1}, \ldots, Q_{d \Delta}$. Recall that $\left|\mathcal{F}_{0}\right|=$ $d$ and so by the pigeonhole principle, there exists a graph $F_{0} \in \mathcal{F}_{0}$ such that at least $\Delta$ paths among $\left\{Q_{1}, \ldots, Q_{\Delta d}\right\}$ are incident to $F_{0}$. Relabelling and keeping the relative ordering, let those paths be $Q_{1}, \ldots, Q_{\Delta}$ connecting $F_{0}$ and $F_{1}^{\prime}, \ldots, F_{\Delta}^{\prime} \in \mathcal{F}^{\prime \prime}$. Let $C_{p+1,0}=F_{0}$ and $C_{p+1, i}=F_{i}^{\prime}$ for each $i \in[\Delta]$.

Now fix $i \in[\Delta]$. By E3 and the definition of $\mathcal{F}^{\prime \prime} \ni F_{i}^{\prime}, B_{G^{\prime}}^{\sqrt[10]{\log n}}\left(V\left(F_{i}^{\prime}\right)\right)$ is disjoint from $Q \backslash V\left(Q_{i}\right)$ and $W$, and furthermore, by Claim 7.4, we can find a connected subgraph $S_{p+1, i} \subseteq B_{G^{\prime}} \sqrt[10]{\log n}\left(V\left(F_{i}^{\prime}\right)\right)$ containing $V\left(F_{i}^{\prime}\right)$, which satisfies D2. Let $v_{p+1, i} \in S_{p+1, i}$ be its first contact point with $Q_{i}$, and let $P_{p+1, i}=Q_{i}-S_{p+1, i} \backslash\left\{v_{p+1, i}\right\}$ be the truncated path. It is routine to check that D1-D4 still hold with the additions of $C_{p+1,0}$, and $C_{p+1, i} \subseteq S_{p+1, i}$ and $P_{p+1, i}, i \leq[\Delta]$. This contradicts the maximality of $p$. Hence, we have $p \geq d$, proving the claim. 


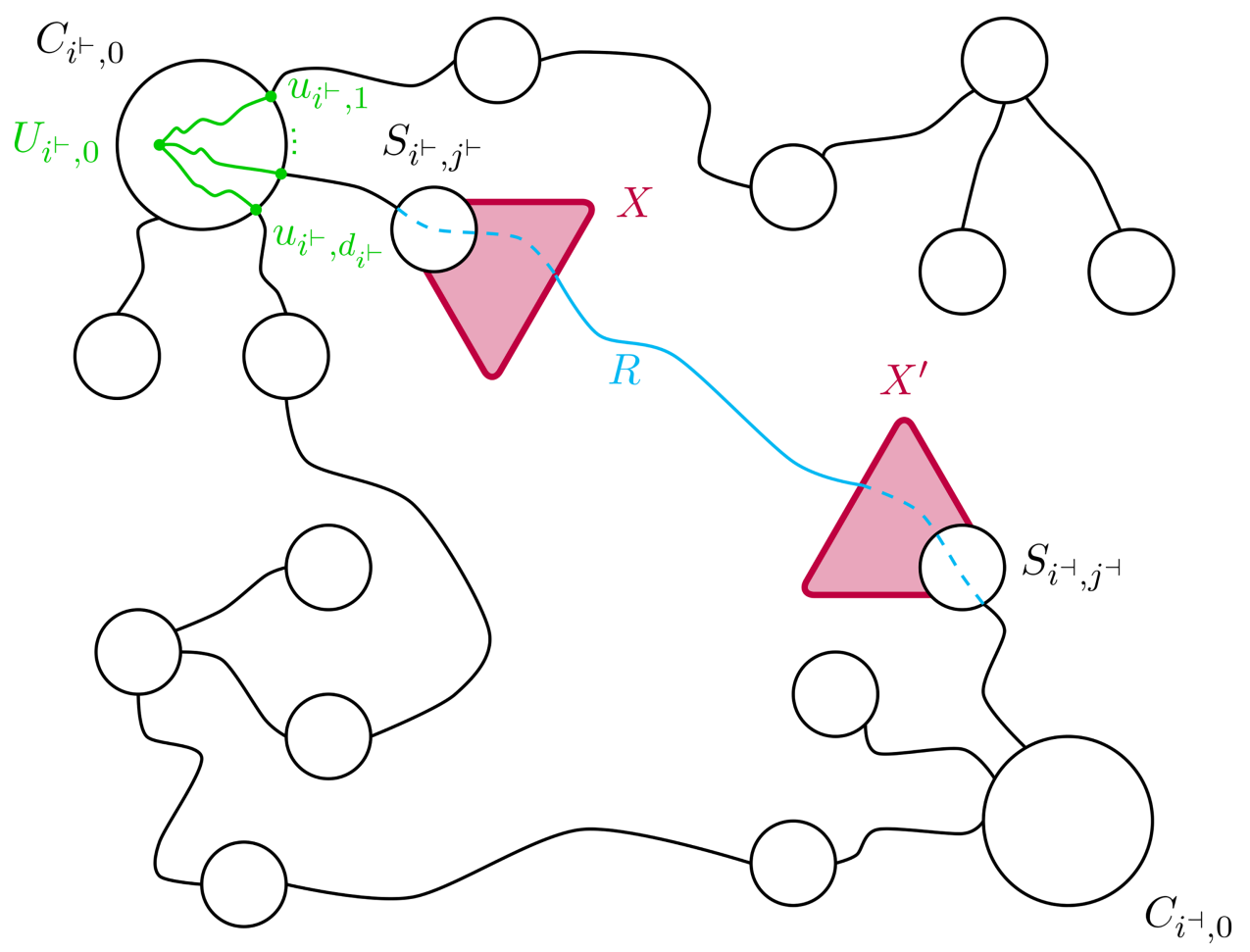

Fig. 6. Connecting nakjis

\subsection{The finishing blow}

It is time now to complete the game: we will wire nakjis together to build an $H$ subdivision.

Enumerate the vertices of $H$ as $x_{1}, \ldots, x_{h}$ and edges of $H$ as $e_{1}=x_{S_{1}} x_{t_{1}}, \ldots, e_{h^{\prime}}=$ $x_{s_{h^{\prime}}} x_{t_{h^{\prime}}}$. Each of the nakjis guaranteed in Claim 7.5 corresponds to a vertex of $H$. We also give an ordering on each nakji's $\Delta$ legs: let $f_{X}, x \in V(H)$, be such that

$$
\left\{f_{X}(e): e \in E(H), x \in e\right\}=\left[d_{H}(x)\right]
$$

Assume we have pairwise disjoint paths $R_{1}, \ldots, R_{\ell}$ for some $0 \leq \ell<h^{\prime}$ satisfying the following for each $i \in[\ell]$, where $\left(i^{\prime}, j^{\prime}\right)=\left(s_{i}, f_{s_{i}}\left(e_{i}\right)\right)$ and $\left(i^{\prime \prime}, j^{\prime \prime}\right)=\left(t_{i}, f_{t_{i}}\left(e_{i}\right)\right)$.

F1 $R_{i}$ is a path between $v_{i^{\prime}, j^{\prime}}$ and $v_{i^{\prime \prime}, j^{\prime \prime}}$ with length at most $10 \mathrm{~m}$.

F2 $R_{i}$ does not intersect with any $C_{i^{*}, 0}$ for $i^{*} \in[h]$ and does not intersect with any $S_{i^{*}, j^{*}} \cup P_{i^{*}, j^{*}}$ for $\left(i^{*}, j^{*}\right) \in[h] \times[\Delta] \backslash\left\{\left(i^{\prime}, j^{\prime}\right),\left(i^{\prime \prime}, j^{\prime \prime}\right)\right\}$. 
Let $\left(i^{\vdash}, j^{\vdash}\right)=\left(s_{\ell+1}, f_{s_{\ell+1}}\left(e_{\ell+1}\right)\right)$ and $\left(i^{\dashv}, j^{\dashv}\right)=\left(t_{\ell+1}, f_{t_{\ell+1}}\left(e_{\ell+1}\right)\right)$. Let

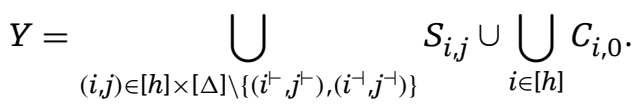

Then by C3, D2 and $d \leq m^{100}$, we have $|Y| \leq(\Delta+1) d \exp (\sqrt[50]{\log n}) \leq \exp (\sqrt[49]{\log n})$. Let

$$
Z=\bigcup_{(i, j) \in[h] \times[\Delta]} V\left(P_{i, j}\right) \cup \bigcup_{i \in[\ell]} V\left(R_{i}\right), \quad X=B_{G^{\prime}-Z}^{\frac{1}{2} \sqrt{\log n}}\left(S_{i^{\vdash}, j^{\vdash}}\right) \quad \text { and } \quad X^{\prime}=B_{G^{\prime}-Z}^{\frac{1}{2} \sqrt{\log n}}\left(S_{i^{\dashv}, j^{\dashv}}\right) .
$$

As $|Z \cup L| \leq d m^{2}$, by $\mathbf{D} 2$ we can apply Proposition 3.5 to $X$ and $X^{\prime}$, with $Z \cup L$ playing the role of $Y$ to obtain that

$$
|X|,\left|X^{\prime}\right| \geq \exp (\sqrt[10]{\log n})
$$

By C2 and D1, D2, we know that $X, X^{\prime}$ does not intersect with $Y$. As $|Y \cup Z \cup L| \leq$ $2 \exp (\sqrt[49]{\log n})<\frac{1}{4} \rho(|Z|)|Z|$ for each $Z \in\left\{X, X^{\prime}\right\}$, by Lemma 3.3, we can find a path $R$ from $X$ to $X^{\prime}$ in $G^{\prime}-Y-Z$ with length at most $m$. As $S_{i, j} \subseteq B{ }_{G^{\prime}}^{\sqrt[10]{\log n}}\left(C_{i, j}\right)$, we know $G\left[S_{i, j}\right]$ is connected due to $\mathbf{D 2}$, and $C_{i, j}$ is an $\left(\varepsilon_{1}, \varepsilon_{2} d\right)$-robust-expander with diameter at most $m$ due to $\mathbf{C} 1$ and $\mathbf{D} 1$, using the definition of $X, X^{\prime}$, we can extend $Z$ inside $X, X^{\prime}$ to get a path from $v_{i^{\vdash}, j^{\vdash}}$ to $v_{i^{\dashv}, j^{\dashv}}$ satisfying F1 and F2 (see Figure 6). Repeating this for each $\ell=0, \ldots, h^{\prime}-1$, we obtain paths $R_{1}, \ldots, R_{h^{\prime}}$ satisfying $\mathbf{F} 1$ and F2.

Note that for each $i \in\left[h^{\prime}\right]$ and $\left(i^{\prime}, j^{\prime}\right)=\left(s_{i}, f_{s_{i}}\left(e_{i}\right)\right),\left(i^{\prime \prime}, j^{\prime \prime}\right)=\left(t_{i}, f_{t_{i}}\left(e_{i}\right)\right)$, the path $R_{i}$ and $P_{i^{\prime}, j^{\prime}} \cup P_{i^{\prime \prime}, j^{\prime \prime}}$ might intersect at their interiors. However, $R_{i} \cup P_{i^{\prime}, j^{\prime}} \cup P_{i^{\prime \prime}, j^{\prime \prime}}$ contains a path $P_{e_{i}}^{*}$ from $v_{i^{\prime}, j^{\prime}}$ to $v_{i^{\prime \prime}, j^{\prime \prime}}$.

Moreover, D3 and F2 imply that each $P_{e_{i}}^{*}$ only intersects $C_{s_{i}, 0}$ and $C_{t_{i}, 0}$ at $u_{i^{\prime}, j^{\prime}}$ and $u_{i^{\prime \prime}, j^{\prime \prime}}$ respectively, and $P_{e_{1}}^{*}, \ldots, P_{e_{h^{\prime}}^{*}}^{*}$ are all pairwise vertex disjoint. Let $P^{*}=\bigcup_{i \in\left[h^{\prime}\right]} V\left(P_{e_{i}}^{*}\right)$.

Now, for each $i \in[h]$, we consider $C_{i, 0}$ and set $d_{i}=d_{H}\left(x_{i}\right)$. Note that $C_{i, 0}$ intersects with $P^{*}$ only at the distinct vertices $u_{i, 1}, \ldots, u_{i, d_{i}}$. We choose a vertex $u_{i, 0} \in C_{i, 0} \backslash$ $\left\{u_{i, 1}, \ldots, u_{i, d_{i}}\right\}$. As $G^{\prime}\left[C_{i, 0}\right]$ is $\varepsilon^{2} v d$-connected with $\varepsilon^{2} v d>\Delta$, we can find a subdivision of $K_{1, d_{i}}$ inside $C_{i, 0}$, where $u_{i, 0}$ corresponds to the centre of the star and the leaves correspond to $u_{i, 1}, \ldots, u_{i, d_{i}}$. These subdivisions of stars together with the paths $P_{e_{i}}^{*}$ all together yield a subdivision of $H$ in $G$. This provides a final contradiction and completes the proof. 


\section{Concluding Remarks}

\subsection{Bounded degree planar graphs as subdivision}

We might ask whether we can in fact guarantee subdivisions rather than minors in Theorem 2.1. Our methods for finding subdivisions only cover bounded-degree graphs, however. So a natural question might be: what is the best constant $c$ such that every graph of average degree $(c+o(1)) t$ contains a subdivision of every bounded-degree planar graph of order $t$ ?

First, it is easy to see that we can achieve $c=5 / 2$ : a planar graph with $t$ vertices has fewer than $3 t$ edges, and any graph has a bipartite subgraph with at least half the edges, so after subdividing at most $3 t / 2$ edges we obtain a bipartite subdivision with at most $5 t / 2$ vertices. Theorem 1.1 then allows us to find a subdivision of this subdivision in any graph of average degree $(5 / 2+o(1)) t$. In fact we can do better.

Lemma 8.1. Any planar graph $H$ on $t$ vertices has a bipartite subdivision with at most $2 t-2$ vertices.

Proof. We may assume $H$ is maximal planar, so every face is a triangle and there are $2 t-4$ faces. Consider the dual graph $H^{*}$. This is a 2-connected 3-regular graph, and so, by Petersen's theorem, has a 1-factor. Each edge of this 1-factor corresponds to an edge of the original graph $H$. We subdivide each of these edges once. Suppose there is an odd cycle $C$ in the original graph $H$. By double-counting edges bordering faces surrounded by $C$, there are an odd number of such faces. Thus, the 1-factor in $H^{*}$ contains an odd number of edges crossing $C$. Thus, we have subdivided an odd number of edges of $C$, so $C$ becomes an even cycle in the subdivision. By exactly the same argument, we can see that any even cycle in $H$ remains even in the subdivision, so the subdivision is bipartite. It has exactly $|H|+\left|H^{*}\right| / 2=2 t-2$ vertices, as required.

Lemma 8.1 is best possible, since in any maximal planar graph we must subdivide at least one edge of each face, and this requires at least $t-2$ extra vertices. Together with Theorem 1.1, it immediately gives the following.

Proposition 8.2. For given $\varepsilon>0$ and $\Delta \in \mathbb{N}$, there exists $d_{0}$ such that if $d \geq d_{0}$ and $H$ is a planar graph with at most $(1-\varepsilon) d$ vertices and $\Delta(H) \leq \Delta$, and $G$ is a graph with average degree at least $2 d$, then $G$ contains a subdivision of $H$. 
Our lower bound from Theorem 2.1 shows only that we cannot improve the constant 2 in Proposition 8.2 below 3/2. However, no similar example will give a stronger lower bound, for the following reason. Any $t$-vertex planar graph $H$ has a bipartite subdivision with at most $3 t / 4$ vertices in one part (to see this, take a largest independent set $X$ and subdivide all edges which do not meet $X$; this is bipartite with one part being $V(H) \backslash X$, which has size at most $3 t / 4$ by the four-colour theorem), and this subdivision is a subgraph of $K_{3 t / 4, n}$ for sufficiently large $n$.

Problem D. What is the right value for the constant in Proposition 8.2? Is it 2, 3/2, or something in between?

Problem E. What can we say about $d_{\top}(H)$ for $k$-degenerate graphs $H$ for general $k \in \mathbb{N}$ ?

\subsection{Better bounds for minor-closed families}

As $\alpha_{2}(F) \geq 2 t / \chi(F)$, writing $\chi(\mathcal{F})=\max \{\chi(F): F \in \mathcal{F}\}$, Theorem 2.2 implies the following Erdoős-Simonovits-Stone type bound for any minor-closed family $\mathcal{F}$ :

$$
d_{\succ}(\mathcal{F}, t) \leq 2\left(1-\frac{1}{\chi(\mathcal{F})}+o(1)\right) t
$$

However, this is in general not tight. It is sharp for the disjoint union of cliques of order $\chi(\mathcal{F})$, if such a graph is in $\mathcal{F}$.

Problem F. Determine $d_{\succ}(\mathcal{F}, t)$ for the nontrivial minor-closed family $\mathcal{F}$. If $\mathcal{F}$ is closed under disjoint union, do we have $d_{\succ}(\mathcal{F}, t)=2(1-1 / \chi(\mathcal{F})+o(1)) t$ ?

In fact, the Hadwiger conjecture would imply $d_{\succ}(\mathcal{F}, t)=2(1-1 / \chi(\mathcal{F})+o(1)) t$ for $\mathcal{F}$ closed under disjoint union. To see this, consider the maximum such that $K_{s} \in \mathcal{F}$. Then, the Hadwidger conjecture would give $\chi(\mathcal{F})=s$. As the disjoint union of $K_{s}$ belongs to $\mathcal{F}$, the above discussion implies $d_{\succ}(\mathcal{F}, t)=2(1-1 / \chi(\mathcal{F})+o(1)) t$. However, a minorclosed family does not have to be closed under disjoint union (e.g., the class of graphs embeddable in a fixed surface other than the plane), and for such families we may have $d_{\succ}(\mathcal{F}, t)<2(1-1 / \chi(\mathcal{F})-c) t$ for some absolute constant $c>0$, as we have seen in Theorem 2.1.

Another interesting question is to see when the upper and lower bounds arising from Theorem 2.2 coincide, which motivates the following problem.

Problem G. For which graphs $G$ do we have $\left|2 \alpha(G)-\alpha_{2}(G)\right|=o(|G|)$ ? For which graphs $G$ do we have $\left|\alpha(G)-\frac{|G|}{\chi(G)}\right|=o(|G|)$ ? Do all minor-closed families $\mathcal{F}$ contain sufficiently large graphs with this property? 
The case $k=6$ of the Hadwiger conjecture, proved by Robertson, Seymour, and Thomas [35], gives the above conclusion for any minor-closed family for which the minimal forbidden minors are all connected and include $K_{6}$. In particular, this applies to the linklessly embeddable graphs discussed in Section 2.2.

Corollary 8.3. The class $\mathcal{L}$ of linklessly embeddable graphs satisfies $d_{\succ}(\mathcal{L}, t)=(8 / 5+$ $o(1)) t$.

Since the $Y \Delta Y$-reducible graphs form a subfamily of $\mathcal{L}$ containing the extremal example $r K_{5}$, the corollary also applies to this class.

\section{Funding}

J.H. and H.L. were supported by the UK Research and Innovation Future Leaders Fellowship MR/S016325/1.

J.K. was supported by the POSCO Science Fellowship of POSCO TJ Park Foundation and by the KAIX Challenge program of KAIST Advanced Institute for Science-X.

\section{Acknowledgement}

We thank O-joung Kwon for bringing our attention to the graph class of bounded expansion. We also thank the referee for the careful readings and suggestions that improve the presentation.

\section{Notes}

1. Nakji means 'long-arm octopus' in Korean.

2. J.H. and H.L. were supported by the UK Research and Innovation Future Leaders Fellowship MR/S016325/1.

3. J.K. was supported by the POSCO Science Fellowship of POSCO TJ Park Foundation and by the KAIX Challenge program of KAIST Advanced Institute for Science-X.

\section{References}

[1] Alon, N., P. Seymour, and R. Thomas. "A separator theorem for nonplanar graphs." J. Amer. Math. Soc. 3, no. 4 (1990): 801-8.

[2] Bollobás, B. and A. Thomason. "Proof of a conjecture of Mader, Erdoős and Hajnal on topological complete subgraphs." European J. Combin. 19, no. 8 (1998): 883-7.

[3] Borodin, O. V. "Solution of the Ringel problem on vertex-face coloring of planar graphs and coloring of 1-planar graphs. (in Russian) Metody Diskret." Analiz. 41 (1984): 12-26.

[4] Böttcher, J., P. Pruessmann, A. Taraz, and A. Würfl. "Bandwidth, expansion, treewidth, separators and universality for bounded-degree graphs." European J. Combin. 31 (2010): 1217-27.

[5] Corrádi, K. and A. Hajnal. "On the maximal number of independent circuits in a graph." Acta Math. Acad. Sci. Hungar. 14 (1963): 423-39. 
[6] Demaine, E. D. and M. Hajiaghayi. "Linearity of grid minors in treewidth with applications through bidimensionality." Combinatorica 28, no. 1 (2008): 19-36.

[7] Djidjev, H. N. “On some properties of nonplanar graphs." C. R. Acad. Bulgare Sci. 37, no. 9 (1984): 1183-4.

[8] Duffin, R. J. “Topology of series-parallel networks." J. Math. Anal. Appl. 10 (1965): 303-18.

[9] Dvořák, Z. and S. Norin. "Strongly sublinear separators and polynomial expansion." SIAM J. Discrete Math. 30, no. 2 (2016): 1095-101.

[10] Erdoős, P. and M. Simonovits. "A limit theorem in graph theory." Studia Sci. Math. Hungar. 1 (1966): 51-7.

[11] Erdoős, P. and A. H. Stone. "On the structure of linear graphs." Bull. Amer. Math. Soc. 52, no. 12 (1946): 1087-91.

[12] Haslegrave, J., J. Kim, and H. Liu. “Extremal density for sparse minors and subdivisions. Online version with appendix." 2020 preprint, arXiv (2012): 02159.

[13] Kawarabayashi, K., Y. Kobayashi, and B. Reed. "The disjoint paths problem in quadratic time." J. Combin. Theory Ser. B 102, no. 2 (2012): 424-35.

[14] Kim, J., H. Liu, M. Sharifzadeh, and K. Staden. "Proof of Komlós's conjecture on Hamiltonian subsets." Proc. London Math. Soc. (3) 115, no. 5 (2017): 974-1013.

[15] Komlós, J., G. N. Sárközy, and E. Szemerédi. "Blow-up lemma." Combinatorica 17 (1997): 09-123.

[16] Komlós, J., A. Shokoufandeh, M. Simonovits, and E. Szemerédi. "The regularity lemma and its applications in graph theory." Theoretical aspects of computer science (Tehran, 2000). 84-112, Lecture Notes in Comput. Sci., 2292. Berlin: Springer, 2002.

[17] Komlós, J. and M. Simonovits. "Szemerédi's regularity lemma and its applications in graph theory." Combinatorics: Paul Erdoös is eighty, Vol. 2 (Keszthely, 1993). 295-352, Bolyai Soc. Math. Stud., 2, János Bolyai Math. Soc., Budapest, 1996.

[18] Komlós, J. and E. Szemerédi. "Topological cliques in graphs." Combin. Probab. Comput. 3 (1994): 247-56.

[19] Komlós, J. and E. Szemerédi. "Topological cliques in graphs II." Combin. Probab. Comput. 5 (1996): 79-90.

[20] Kostochka, A. V. "Lower bound of the Hadwiger number of graphs by their average degree." Combinatorica 4, no. 4 (1984): 307-16.

[21] Liu, H. and R. Montgomery. "A proof of Mader's conjecture on large clique subdivisions in $C_{4}$-free graphs." J. Lond. Math. Soc 95, no. 1 (2017): 203-22.

[22] Mader, W. "Homomorphiesätze für Graphen. (in German)." Math. Ann. 178 (1968): 154-68.

[23] Mader, W. “Existenz $n$-fach zusammenhängender Teilgraphen in Graphen genügend grosser Kantendichte." (in German) Abh. Math. Sem. Univ. Hamburg 37 (1972): 86-97.

[24] Messuti, S., V. Rödl, and M. Schacht. "Packing minor-closed families of graphs into complete graphs." J. Combin. Theory Ser. B 119 (2016): 245-65.

[25] Miller, G. L., S.-H. Teng, W. Thurston, and S. A. Vavasis. "Separators for sphere-packings and nearest neighbor graphs." J. ACM 44, no. 1 (1997): 1-29. 
[26] Myers, J. S. and A. Thomason. "The extremal function for noncomplete minors." ombinatorica 25, no. 6 (2005): 725-53.

[27] Nešetřil, J. and P. Ossona de Mendez. "Grad and classes with bounded expansion. I. Decompositions." European J. Combin. 29, no. 3 (2008): 760-76.

[28] Nešetřil, J., P. Ossona de Mendez, and D. Wood. "Characterisations and examples of graph classes with bounded expansion." European J. Combin. 33, no. 3 (2012): 350-73.

[29] Norin, S., L. Postle, and Z.-X. Song. "Breaking the degeneracy barrier for coloring graphs with no $K_{t}$ minor." (2019) preprint, arXiv:1910.09378.

[30] L "Postle, An even better Density Increment Theorem and its application to Hadwiger's Conjecture." 2020 preprint, arXiv (2006): 14945.

[31] Reed, B. and D. Wood. "Forcing a sparse minor." Combin. Probab. Comput. 25 (2016): 300-22.

[32] Robertson, N. and P. D. Seymour. "Graph minors. V. Excluding a planar graph." J. Combin. Theory Ser. B 41, no. 1 (1986): 92-114.

[33] Robertson, N. and P. D. Seymour. "Graph minors. XIII. The disjoint paths problem." J. Combin. Theory Ser. B 63, no. 1 (1995): 65-110.

[34] Robertson, N. and P. D. Seymour. "Graph Minors. XX. Wagner's conjecture." J. Combin. Theory Ser. B 92, no. 2 (2004): 325-57.

[35] Robertson, N., P. D. Seymour, and R. Thomas. "Hadwiger's conjecture for $\mathrm{K}_{6}$-free graphs." Combinatorica 13, no. 3 (1993): 279-361.

[36] Robertson, N., P. D. Seymour, and R. Thomas. "Quickly excluding a planar graph." J. Combin. Theory Ser. B 62, no. 2 (1994): 323-48.

[37] Robertson, N., P. D. Seymour, and R. Thomas. "Sachs' linkless embedding conjecture." J. Combin. Theory Ser. B 64, no. 2 (1995): 185-227.

[38] Thomason, A. "An extremal function for contractions of graphs." Math. Proc. Cambridge Phil. Soc. 95 (1984): 261-5.

[39] Thomason, A. "The extremal function for complete minors." J. Combinatorial Theory Ser. B 81 (2001): 318-38.

[40] Voss, H. and C. Zuluaga. "Maximale gerade und ungerade Kreise in Graphen. I. (in German)." Wiss. Z. Techn. Hochsch. Ilmenau 23 (1977): 57-70.

[41] Yu, Y. "More forbidden minors for Wye-Delta-Wye reducibility." Electron. J. Combin. 13, no. 1 (2006) paper no. 7, 15 pp. 